\title{
Azobenzene-Equipped Covalent Organic Framework: Light-Operated Reservoir
}

Gobinda Das, ${ }^{\text {aः }}$ Thirumurugan Prakasam ${ }^{\text {a" }}$ Matthew A. Addicoat, ${ }^{\mathrm{b}}$ Sudhir Kumar Sharma, ${ }^{\mathrm{c}}$ Florent Ravaux, ${ }^{\mathrm{d}}$ Renny Mathew, ${ }^{\mathrm{a}}$ Maria Baias, ${ }^{\mathrm{a}}$ Ramesh Jagannathan, ${ }^{\mathrm{c}}$ Mark A. Olson, ${ }^{\mathrm{e}}$ Ali Trabolsi*a

${ }^{a}$ Chemistry Program, New York University Abu Dhabi (NYUAD), Saadiyat Island, United Arab Emirates

${ }^{\mathrm{b}}$ School of Science and Technology, Nottingham Trent University Clifton Lane, NG11 8NS Nottingham (UK)

${ }^{\mathrm{c}}$ Engineering Division, New York University Abu Dhabi (NYUAD), United Arab Emirates

d Electrical and Computer Engineering Department, Khalifa University, 127788 Abu Dhabi, United Arab Emirates

${ }^{\mathrm{e}}$ School of Pharmaceutical Science and Technology, Tianjin University, Tianjin, P. R. China

\#Equal contribution

\section{$\underline{\text { Supporting information }}$}




\section{Table of Contents}

1. General Material and Instrumentation Methods

2. Synthesis of Azo-DFP ligand and TTA-AzoDFP

2.1. Synthesis of $(E)$-1-(4-iodophenyl)-2-phenyldiazene (2b)

2.2. Synthesis of (E)-1-phenyl-2-(4-(4,4,5,5-tetramethyl-1,3,2-dioxaborolan-2-

yl)phenyl)diazene (2c)

2.3. Synthesis of (E)-4-(4-(phenyldiazenyl)phenyl)pyridine-2,6-dicarbaldehyde (AzoDFP)

2.4 Synthesis of TTA-AzoDFP

3. FT-IR analysis of TTA-AzoDFP and its precursors

4. Solid state ${ }^{13} \mathrm{C}$ cross-polarization magic-angle spinning NMR spectral analysis

5. Thermogravimertic analysis (TGA) of TTA-AzoDFP and its precursors

6. SEM and TEM analysis of TTA-AzoDFP

7. AFM analysis of TTA-AzoDFP

8. Computational method

9. Comparison of PXRD pattern between azo benzene free TTA-DFP and trans-TTAAzoDFP TGA analysis of TTA-AzoDFP

10. Azo-DFP monomer: ${ }^{1} \mathrm{H}$ NMR spectral analysis before and after UV-light irradiation in solution

11. Time dependent UV-Vis study of TTA-AzoDFP

12. Photo-stability of TTA-AzoDFP

13. Luminescence study of Azo-DFP

14. BET analysis of TTA-AzoDFP COF

15. BET analysis of TTA-DFP COF

16. Solid state ${ }^{13} \mathrm{C}$ cross-polarization magic-angle spinning NMR spectral analysis before and after UV-light irradiation

17. DLS experiment of the photo-isomerization of trans-TTA-AzoDFP

18. Light-triggered capture and release of Rhodamine $B$

19. BET analysis after loading of $\mathrm{RhB}$

20. Control experiment for contact angle measurement and the cargo loading in isostructural TTA-DFP COF

21. UV-Vis experiment of cargo release in response to UV-light

22. Control UV-Vis experiment of cargo release in response to heat

23. BET analysis after regeneration

24. References 


\section{General Material and Instrumentation Methods}

All reagents and starting materials were purchased from Sigma-Aldrich and used without further purification. The 4-bromo pyridine-2,6-dicarbaldehyde (1d $)^{1,2}$ and (E)-1-phenyl-2-(4-(4,4,5,5tetramethyl-1,3,2-dioxaborolan-2-yl)phenyl)diazene $(\mathbf{2 c})^{3}$ were synthesized as previously reported. Thin-layer chromatography (TLC) was performed on silica gel 60 F254 (E. Merck). The plates were inspected with UV light. Column chromatography was performed on silica gel 60F (Merck 9385, 0.040-0.063 mm). Routine nuclear magnetic resonance (NMR) spectra were recorded at $25^{\circ} \mathrm{C}$ on a Bruker Avance III spectrometer, with working frequencies of $500 \mathrm{MHz}$ for ${ }^{1} \mathrm{H}$, and $125.0 \mathrm{MHz}$ for ${ }^{13} \mathrm{C}$ nuclei. All chemical shifts are reported in ppm relative to the signals corresponding to the residual non-deuterated solvents $\left(\mathrm{CDCl}_{3}=7.26 \mathrm{ppm}\right) .{ }^{4,5} \mathrm{All}{ }^{13} \mathrm{C}$ spectra were recorded with the simultaneous decoupling of proton nuclei. Coupling constant values $(J)$ are given in hertz $(\mathrm{Hz})$. The multiplicity of the proton spectrum is abbreviated in the following way: s (singlet), d (doublet), dd (doublet of doublets), t (triplet), q (quartet), qt (quintet), sx (sextet), $\mathrm{m}$ (multiplet) and a wide signal is preceded by br (broad). High resolution mass spectrometry (HRMS) analyses were performed using an Agilent 6540 UHA Accurate Mass Q-TOF / LC - MS-spectrometer in the positive mode and an acetonitrile/water used a gradient in C18 column. Fourier transform infrared (FTIR) studies were carried out on the Agilent 670-IR spectrometer. The contact angles were measured using dataphysics OCA 15EC instrument. Thermogravimetric analysis (TGA) was performed on TA SDT Q600. Scanning electron microscopy (SEM) images were obtained from FEI Quanta 450FEG. The topography of the trans-TTA-AzoDFP and cis-TTA-AzoDFP was analyzed by dynamic atomic force microscopy (5500 Atomic Force Microscope; Keysight Technologies Inc., Santa Rosa, CA), the contact angle was measured by coating an ethanolic dispersion of the COF powder on a smooth flat glass surface by homogeneous drop casting technique to make the surface smooth as much as possible. We acquired topography, phase and amplitude scans simultaneously. Silicon cantilevers (Nanosensors $^{\mathrm{TM}}$, Neuchatel, Switzerland) with resonant frequencies of $250-300 \mathrm{kHz}$ and force constants of $100-130 \mathrm{Nm}^{-1}$ were used. The set point value was kept at $2.5 \mathrm{~V}$. AFM scans were collected at 1024 points/lines with scan speed of 0.20 at fixed scan angle of $0^{\circ}$. Scan artifacts were minimized by acquiring a typical scan at an angle of $90^{\circ}$ under identical image acquisition parameters. We used GwyddionTM free software (version 2.47), SPM data visualization and analysis tool for post-processing the AFM scans. Size and morphology of the COF material was determined with a TEM (FEI-Titan 300) microscope. Samples were prepared on a carbon-coated copper grid. A drop of dispersed TTA-AzoDFP network was spotted on the grid and allowed to dry overnight. Surface area measurements were conducted on a Micromeritics 3Flex gas sorption analyzer. Samples $(20-50 \mathrm{mg})$ were degassed at $85{ }^{\circ} \mathrm{C}$ for 24 $\mathrm{h}$ and then backfilled with $\mathrm{N}_{2}$. Adsorption isotherms were generated by incremental exposure to ultrahigh-purity nitrogen up to $1 \mathrm{~atm}$ in a liquid nitrogen bath, and surface parameters were determined using BET adsorption models included in the instrument software (Micromeritics ASAP 2020 V4.00). Powder X-ray diffraction (PXRD) measurements were carried out using the PANalyticalX'Pert PRO MP X-ray diffractometer consisting of a focusing elliptical mirror and 
a fast-high resolution detector (PIXCEL) with the radiation wavelength of $0.15418 \mathrm{~nm}$. All solidstate NMR experiments were carried out on a Bruker Avance-HD $600 \mathrm{MHz}$ spectrometer operating at a static field of $14.1 \mathrm{~T}$, resonating at $150.0 \mathrm{MHz}$ for ${ }^{13} \mathrm{C}$, using a $3.2 \mathrm{~mm}$ triple resonance MAS probe. UV-Vis studies were carried out on the Cary $5000 \mathrm{UV}-\mathrm{Vis}-\mathrm{NIR}$ spectrophotometer using a Hellma Quartz cuvette with $1 \mathrm{~cm}$ length. For photoisomerisation, the samples were irradiated with an LED lamp (OptoLED, Cairn Research Ltd.) using a $365 \mathrm{~nm}$ light (average power, 15-20 $\mathrm{mW}$ ). The light source was placed at a distance of $\sim 5 \mathrm{~cm}$ from the sample.

\section{Synthesis of Azo-DFP ligand and TTA-AzoDFP}

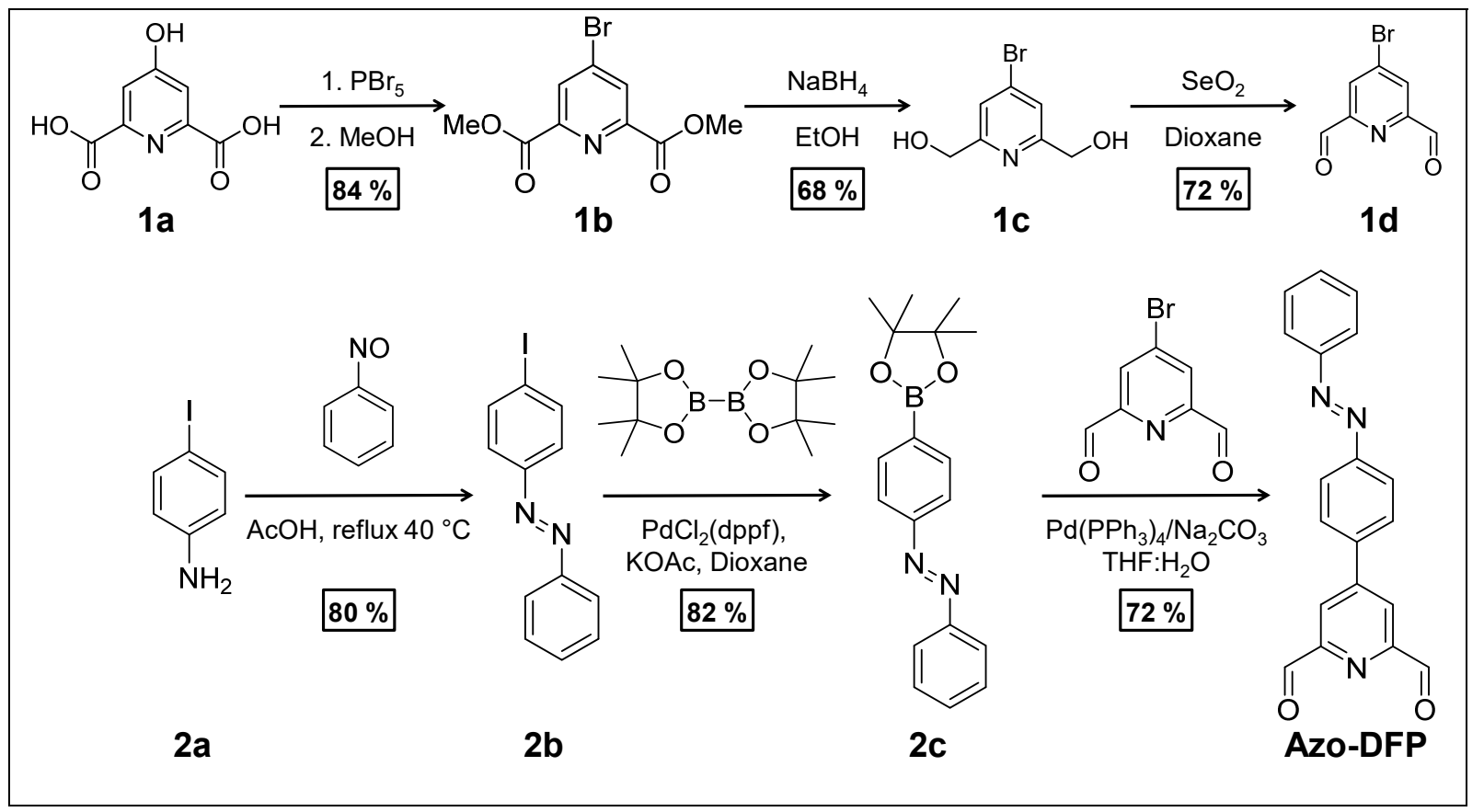

Scheme S1: Synthetic route of 4-azo benzene substituted 2, 6-diformyl pyridine (Azo-DFP)

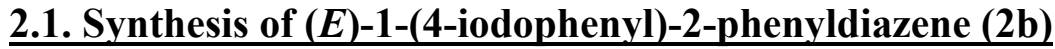

Compound $\mathbf{2 b}$ was prepared according to the literature with slight modification. ${ }^{3} 4$-iodoaniline $(1.7 \mathrm{~g}, 7.75 \mathrm{mmol})$ was added to nitrosobenzene $(1.0 \mathrm{~g}, 9.3 \mathrm{mmol})$ in concentrated acetic acid $(65 \mathrm{~mL})$ in a $250 \mathrm{~mL}$ round bottom flask. The reaction mixture was heated at $40{ }^{\circ} \mathrm{C}$ overnight with uniform stirring. After the reaction completion, distilled water $(500 \mathrm{~mL})$ was added to the reaction mixture and the obtained precipitate was filtered and washed with $500 \mathrm{~mL}$ of water. The crude product was recrystallized from hot ethanol to give compound $\mathbf{2 b}$ in $80 \%$ yield as orange crystals. ${ }^{1} \mathrm{H}$ NMR $\left(500 \mathrm{MHz}, \mathrm{CDCl}_{3}, 25^{\circ} \mathrm{C}\right): \delta$ 7.51-7.56 (m, 3H, Ar- $\left.H\right), 7.67(\mathrm{~d}, J=8.7 \mathrm{~Hz}$, 2H, Ar-H), 7.87-7.90 (m, 2H, Ar-H), 7.93-7.95 (m, 2H, Ar- H); ${ }^{13} \mathrm{C}$ NMR (125 MHz, CDCl, 25 $\left.{ }^{\circ} \mathrm{C}\right): \delta 97.6,122.9,124.5,129.2,131.4,138.4,151.9,152.5$. 


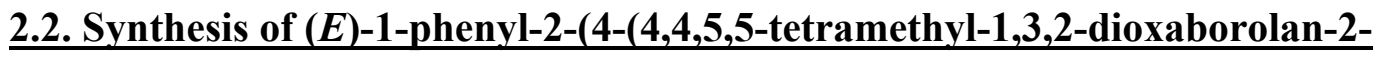 yl)phenyl)diazene (2c)}

(E)-1-(4-iodophenyl)-2-phenyldiazene (2b) $(708 \mathrm{mg}, 2.3 \mathrm{mmol})$ was stirred with a mixture of potassium acetate $(500 \mathrm{mg}, 5.1 \mathrm{mmol})$ and bis(pinacolato)diboron $(812 \mathrm{mg}, 3.2 \mathrm{mmol})$ in dioxane $(20 \mathrm{~mL})$ under argon. After $15 \mathrm{~min}, \mathrm{PdCl}_{2}(\mathrm{dppf})_{2}(220 \mathrm{mg}, 0.3 \mathrm{mmol})$ was added and the suspension was stirred overnight at $90{ }^{\circ} \mathrm{C}$. The reaction mixture was concentrated to dryness after the completion of the reaction and the crude azo-boronic ester was purified by column chromatography $\left(\mathrm{SiO}_{2}\right.$ : EtOAc/n-hexane (1/20)). Compound $2 \mathrm{c}$ was isolated in $82 \%$ yield as orange solid. ${ }^{1} \mathrm{H}$ NMR $\left(500 \mathrm{MHz}, \mathrm{CDCl}_{3}, 25{ }^{\circ} \mathrm{C}\right): \delta 1.40(\mathrm{~s}, 12 \mathrm{H},-\mathrm{CH}), 7.51-7.57(\mathrm{~m}, 3 \mathrm{H}, \mathrm{Ar}-$ H), 7.92-8.00 (m, 6H, Ar-H); ${ }^{13} \mathrm{C}$ NMR (125 MHz, $\left.\mathrm{CDCl}_{3}, 25{ }^{\circ} \mathrm{C}\right): \delta 24.9,84.1,121.9,123.0$, 129.1, 131.2, 135.7, 152.7, 154.4.

\subsection{Synthesis of $(E)-4-(4-($ phenyldiazenyl)phenyl)pyridine-2,6-dicarbaldehyde (Azo-DFP)}

4-Bromo-2,6-diformylpyridine (1d) (500 mg, $2.34 \mathrm{mmol}$ ) was added to compound 2c (723 $\mathrm{mg}$, $2.34 \mathrm{mmol})$ and $\mathrm{Pd}\left(\mathrm{PPh}_{3}\right)_{4}(271 \mathrm{mg}, 0.10 \mathrm{mmol})$ in $100 \mathrm{~mL}$ three neck round-bottom flask under argon atmosphere. $15 \mathrm{~mL}$ of degassed 1:1 THF/H2 $\mathrm{O}$ mixed solvent containing $\mathrm{NaHCO}_{3}(20 \%$ solution) was added to the reaction mixture. The reaction mixture was then refluxed at $85^{\circ} \mathrm{C}$ for $15 \mathrm{~h}$ under an inert atmosphere of argon. After the reaction completion, $20 \mathrm{~mL}$ of water was added to the reaction mixture and aqueous solution was extracted with dichloromethane $(3 \times 20 \mathrm{ml})$. The combined organic layer was dried with magnesium sulphate, filtered and concentrated to dryness. The resulting red solid was purified by flash column chromatography $\left[\mathrm{SiO}_{2}: \mathrm{CH}_{2} \mathrm{Cl}_{2}\right]$ to yield Azo-DFP as a dark red color in $72 \%$ yield. ${ }^{1} \mathrm{H} \mathrm{NMR}\left(500 \mathrm{MHz}, \mathrm{CDCl}_{3}\right.$, $\left.25^{\circ} \mathrm{C}\right): \delta$ 7.54-7.59 (m, 3H, Ar-H), 7.93-7.96 (m, 2H, Ar-H), 7.98-8.02 (m, 2H, Ar-H), 8.10-8.13 (m, 2H, Ar-H), 8.49 (s, 2H, Ar-H), 10.27 (s, 2H, Ar-CH=O); ${ }^{13} \mathrm{C} \mathrm{NMR}\left(75 \mathrm{MHz}, \mathrm{CDCl}_{3}, 25{ }^{\circ} \mathrm{C}\right)$ : $\delta 122.8,123.1,123.9,128.1,129.2,131.7,138.0,150.2,152.6,153.5,153.8,192.4$; MS (ESIHRMS): m/z Calcd for $\left(\mathrm{C}_{19} \mathrm{H}_{14} \mathrm{~N}_{3} \mathrm{O}_{2}\right)^{+}: 316.1086(\mathrm{M}+\mathrm{H})^{+}$, found: $316.1086,(\mathrm{M}+\mathrm{H})^{+}$

\subsection{Synthesis of TTA-AzoDFP}

4, 4', 4"-(1,3,5-triazine-2,4,6-triyl)trianiline (TTA) was synthesized according to the published procedure with no modifications. ${ }^{6}$ A $25 \mathrm{~mL}$ high-pressure flask was charged with 1,3,5-tris(4aminophenyl) benzene (TTA, $21 \mathrm{mg}, 0.06 \mathrm{mmol}, 2$ equiv) and (E)-4-(4(phenyldiazenyl)phenyl)pyridine-2,6-dicarbaldehyde (Azo-DFP, $106.2 \mathrm{mg}, 0.09 \mathrm{mmol}, 3$ 
equiv). Anhydrous 1,4-dioxane $(3.0 \mathrm{~mL})$ was added and the resulting mixture was sonicated for 1 minute. An aqueous solution of acetic acid $(0.5 \mathrm{~mL}, 3.0 \mathrm{M})$ was added, resulting in immediate formation of a yellow precipitate. The resulting mixture was kept in oven at $120^{\circ} \mathrm{C}$ for 1 hour and subsequently cooled to room temperature. The precipitate was collected by centrifugation and washed with anhydrous ethanol five times and with water twice. The powder was dried at $120^{\circ} \mathrm{C}$ under vacuum overnight to yield the yellow colored product.

\section{FT-IR analysis of TTA-AzoDFP and its precursors}

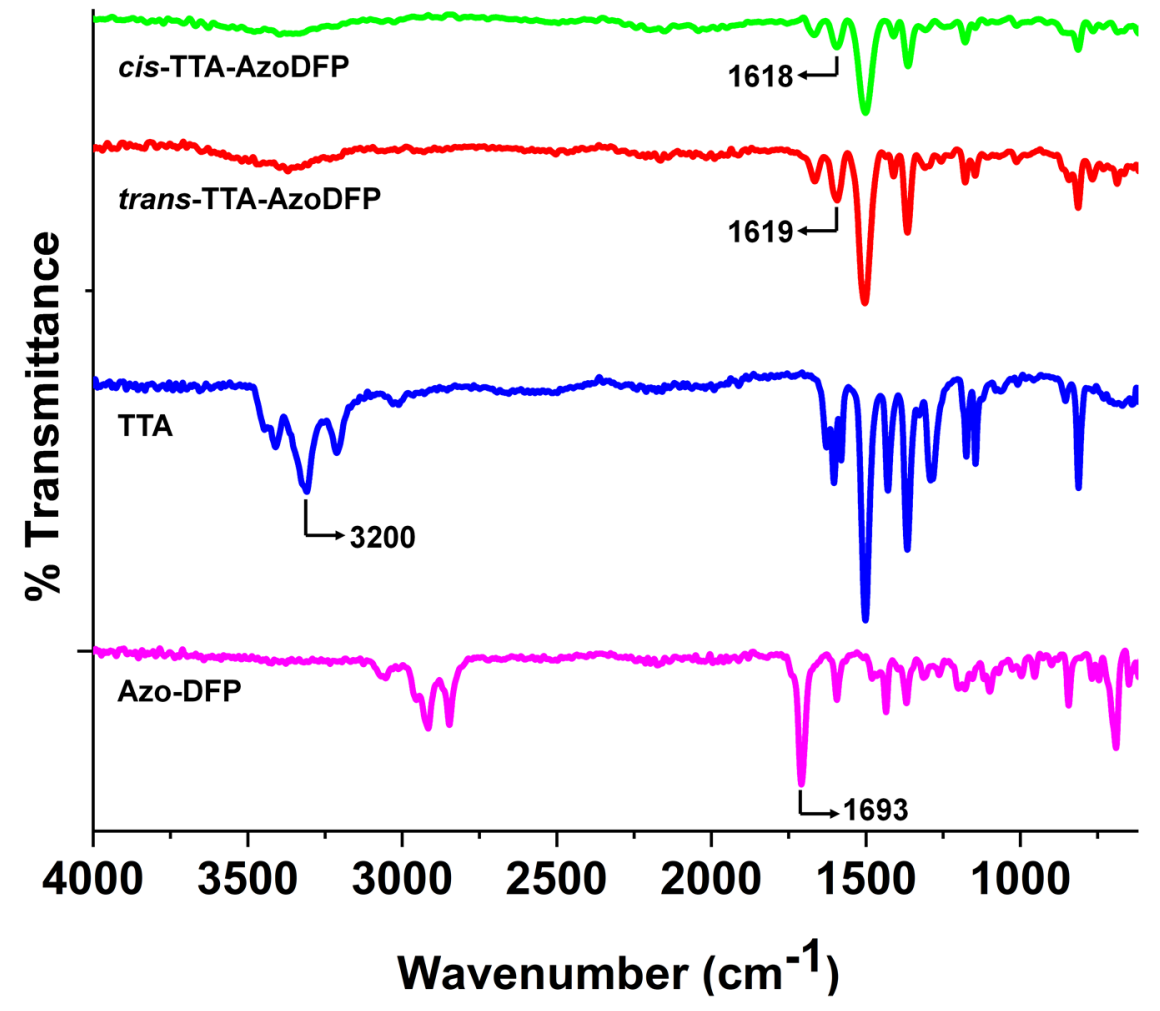

Figure S1: Stacked FTIR spectra of the as-synthesized trans-TTA-AzoDFP (red line), cis-TTAAzoDFP (green line) irradiated with UV-light (365 nm, $30 \mathrm{~min}$ ), 4, 4', 4"-(1,3,5-triazine-2,4,6triyl)trianiline (TTA, blue line) and (E)-4-(4-(phenyldiazenyl)phenyl)pyridine-2,6dicarbaldehyde (Azo-DFP) (purple line). 


\section{$\underline{\text { 4. Solid state }{ }^{13} \mathrm{C} \text { cross-polarization magic-angle spinning NMR spectral analysis }}$}

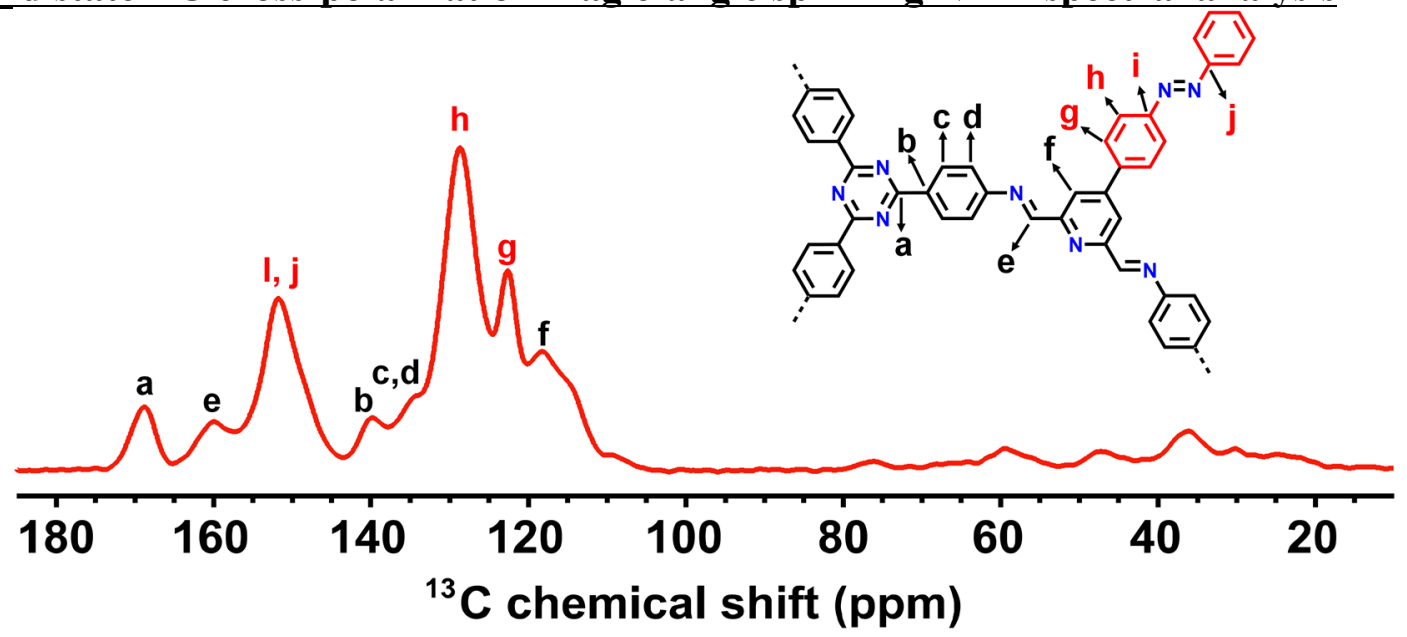

Figure S2: Solid-state ${ }^{13} \mathrm{C}$ CP-MAS NMR analysis of the as-synthesized trans-TTA-AzoDFP. Samples in the form of a fine powder were packed into a $3.2 \mathrm{~mm}$ O.D. zirconia rotor and spun at $24 \mathrm{kHz} .{ }^{1} \mathrm{H}-{ }^{13} \mathrm{C} \mathrm{CP} / \mathrm{MAS}$ measurements were performed using a standard Ramped-Amplitude Cross-Polarization (RAMPCP) pulse sequence.

\section{Thermogravimertic analysis (TGA) of TTA-AzoDFP and its precursors}

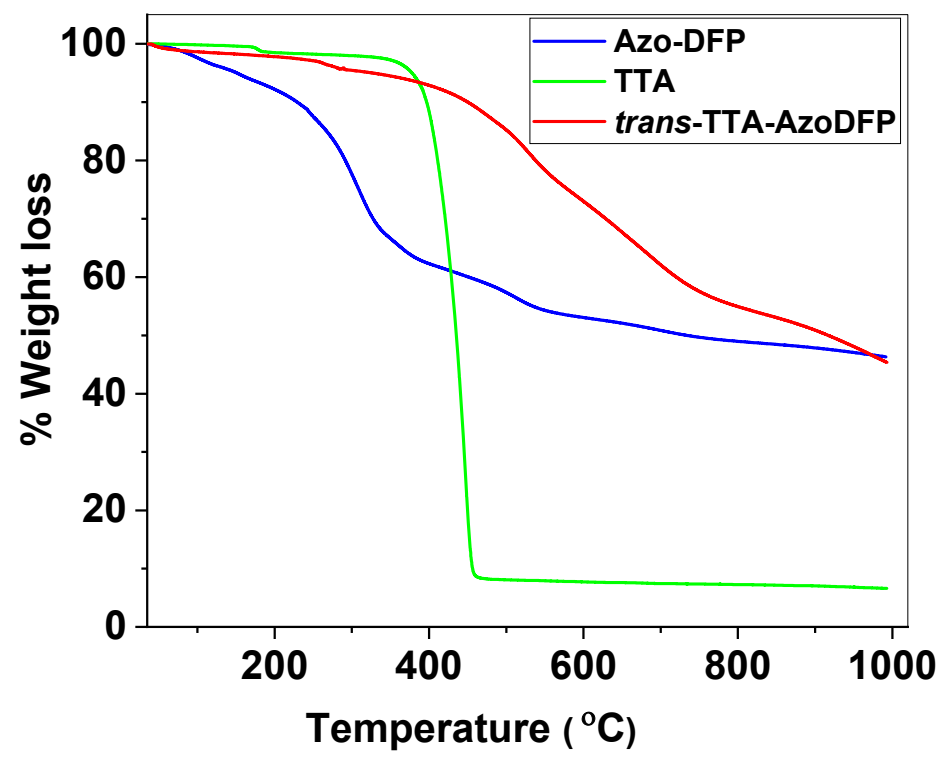

Figure S3: TGA profiles of Azo-DFP (blue line), TTA (green line) and the as-synthesized transTTA-AzoDFP COF (red line), showing the better thermal stability of the COF compared to its precursors. 


\section{SEM and TEM analysis of TTA-AzoDFP}
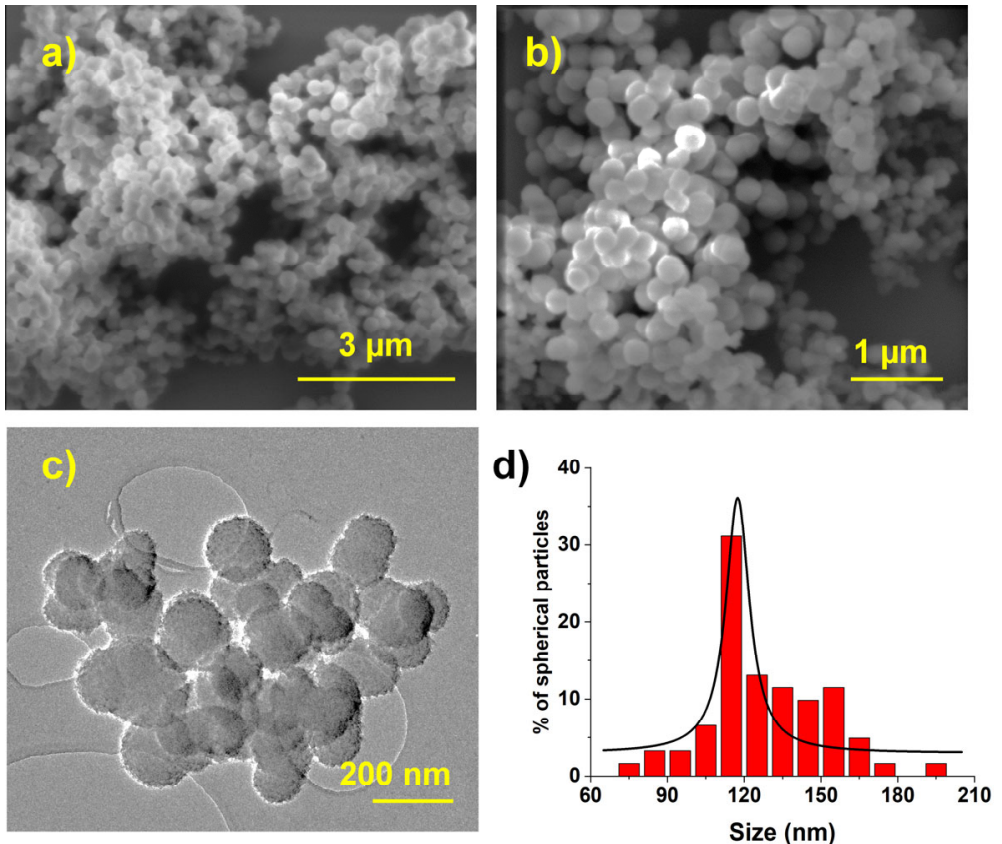

Figure S4: SEM (a, b) and TEM c) images of trans-TTA-AzoDFP; d) Size distribution of the spherical as-synthesized trans-TTA-AzoDFP.

\section{AFM analysis of TTA-AzoDFP}
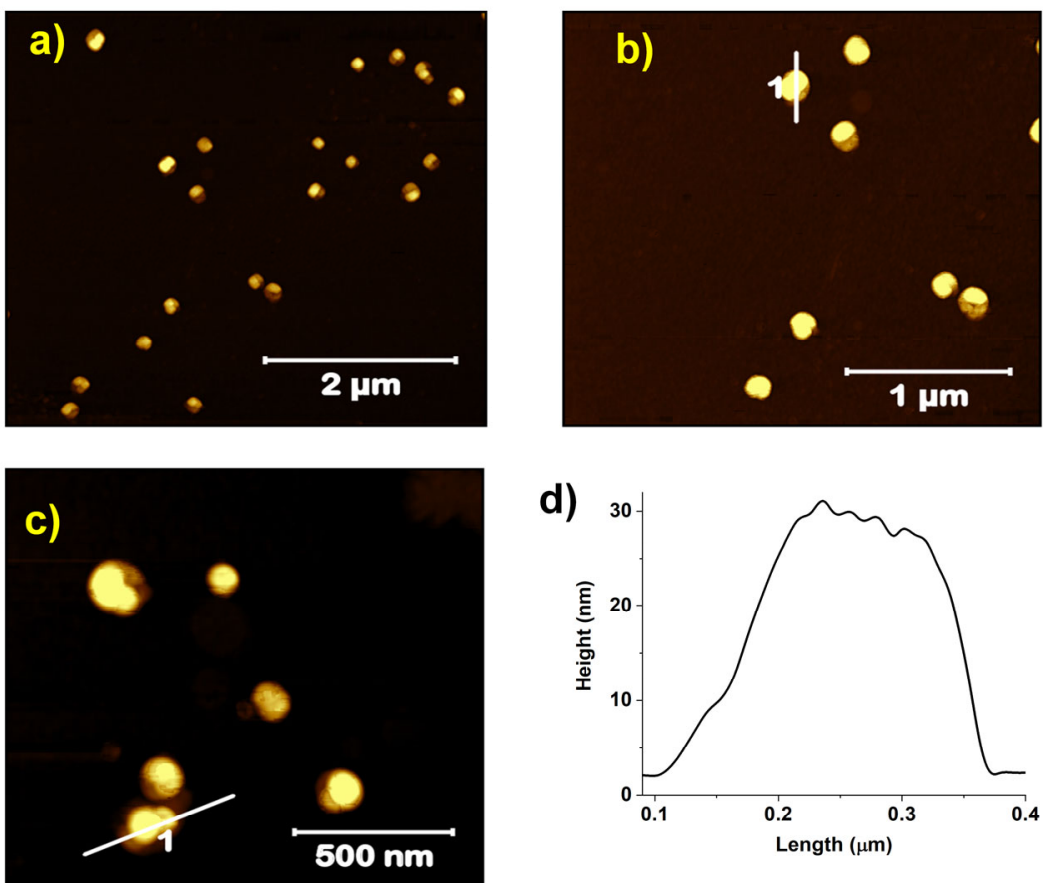

d)

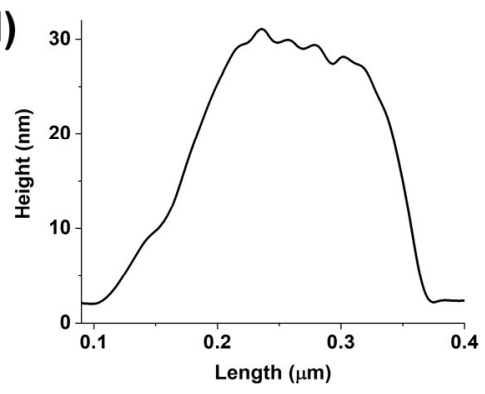

Figure S5: AFM micrographs (a-c) and Height profile (d) of the spherical trans-TTAAzoDFP. 


\section{Computational method}

The atomic positions and cell parameters of the TTA-AzoDFP COF were optimized using the SelfConsistent-Charge Density-Functional Tight-Binding (SCC-DFTB) method as implemented in DFTB+ 17.1. All atoms were described using the 3ob-3-1 parameter set $^{7,8}$ and Lennard-Jones dispersion. In order to minimize the effect of the charged framework and "free" chloride ions, all optimizations used a $2 \times 2$ unit cell. Layer stacking is affected by the Coulomb repulsion between the partial atomic charges in adjacent layers. ${ }^{9}$ Hence, from the optimized monolayer, several stacking possibilities were calculated, including AA (eclipsed) and slipped AA stacking.

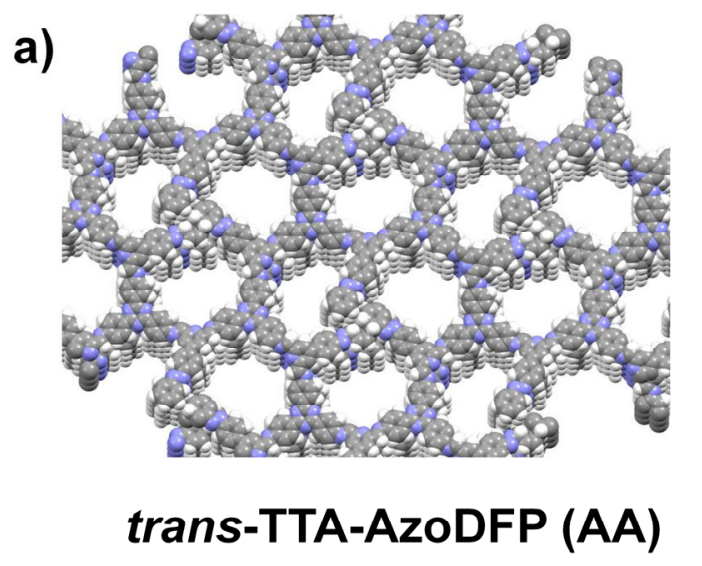

Total energy: -485.763293 a.u.

LJ energy: 1.5405 a.u.

Per layer stabilization energy: - 67.41 (kcal/mol)

HOMO-LUMO gap: $1.030 \mathrm{eV}$

b)

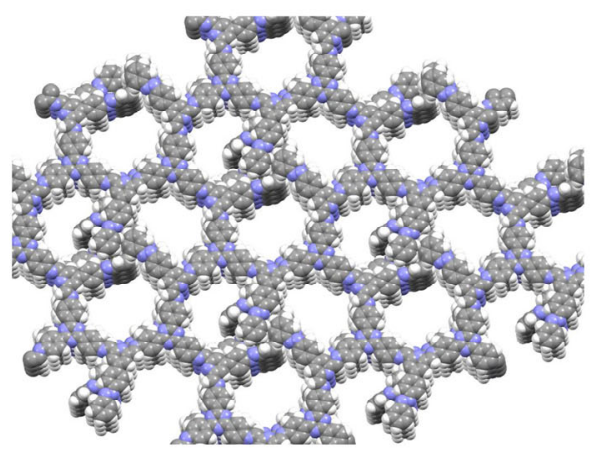

Total energy: - 485.953302 a.u.

LJ energy: 1.4994 a.u.

Per layer stabilization energy: -127.02 (kcal/mol) HOMO-LUMO gap: $1.763 \mathrm{eV}$

\section{trans-TTA-AzoDFP (slip-AA)}

Figure S6: DFT optimized structures of trans-TTA-AzoDFP with AA (a) and slip-AA (b) stacking. The predicted slipped structure has the lowest energy, while maintaining agreement with the experimental PXRD pattern. 

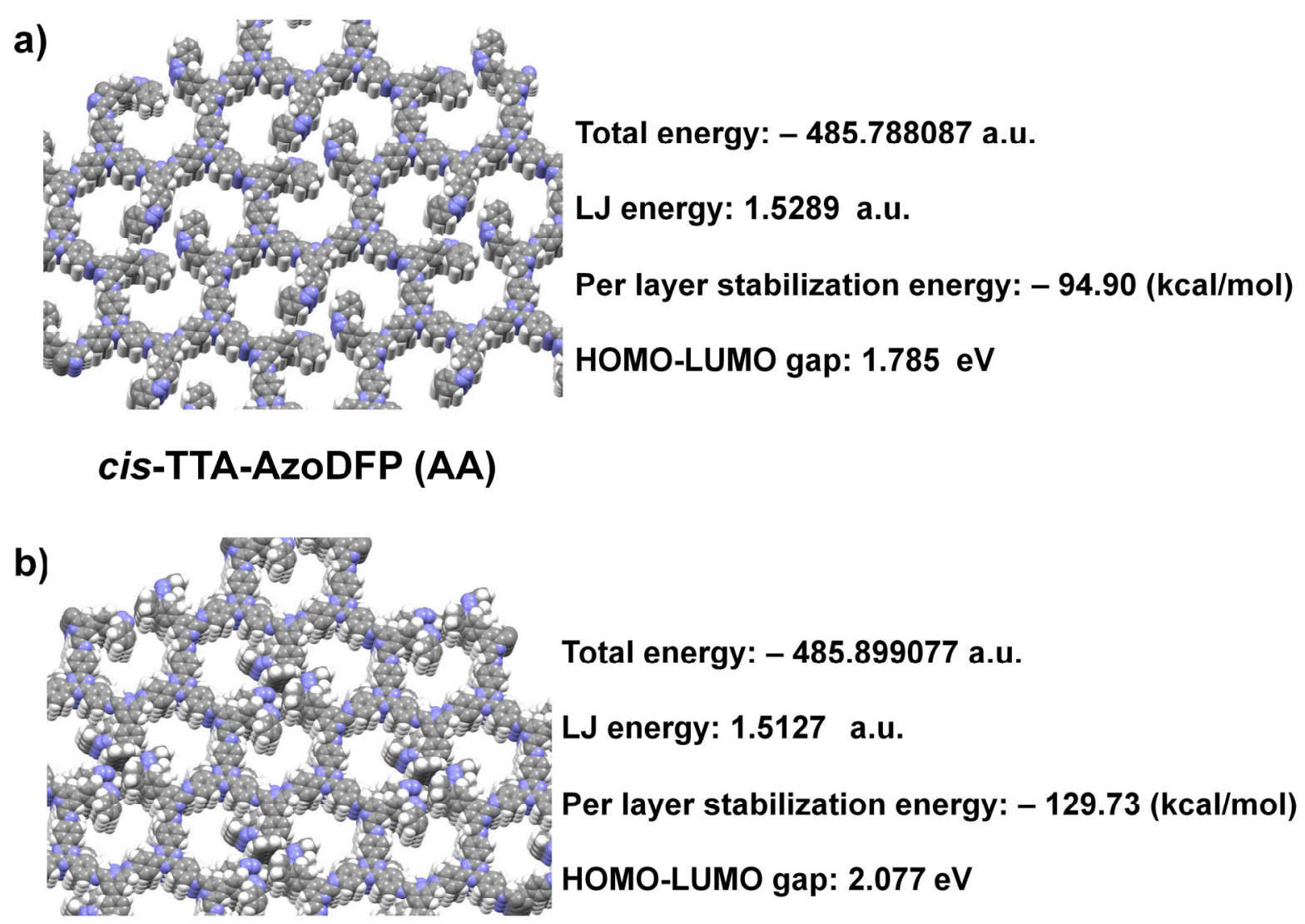
cis-TTA-AzoDFP (slip-AA)

Figure S7: DFT optimized structures of cis-TTA-AzoDFP with AA (a) and slip-AA (b) stacking. The predicted slipped structure has the lowest energy, while maintaining agreement with the experimental PXRD pattern. 
a)



b)
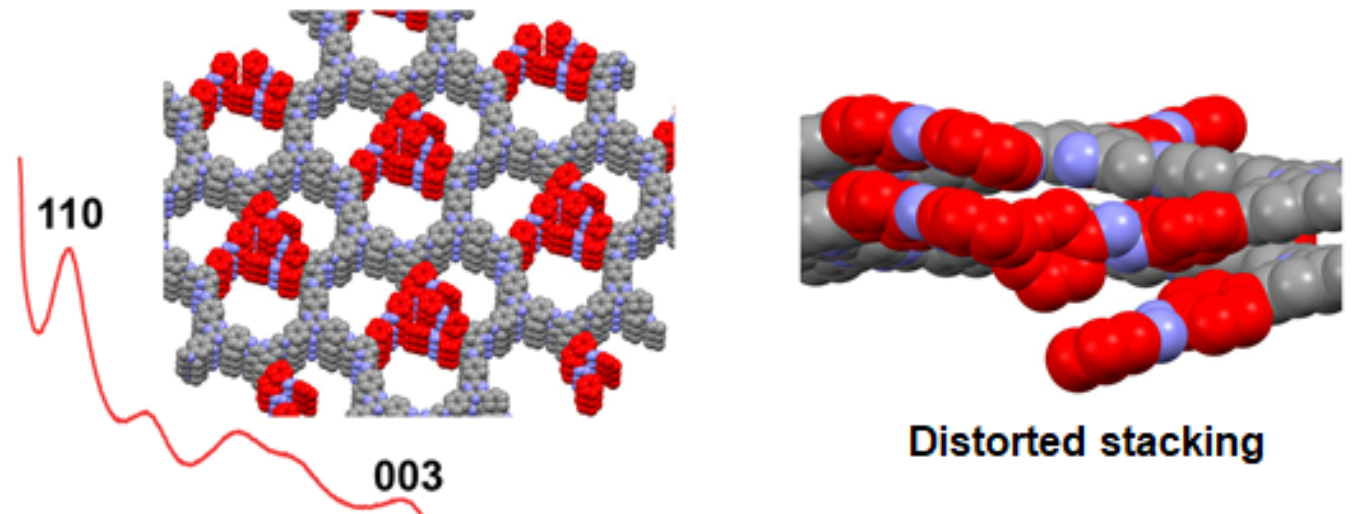

Distorted stacking

\section{Undistorted stacking}

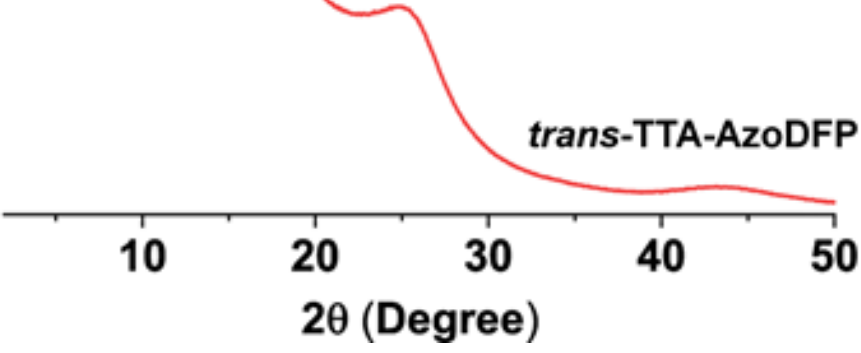

Figure S8: PXRD patterns of a) azobenzene free TTA-DFP and b) as-synthesized trans-TTA-AzoDFP COF prepared under the same conditions. The broad PXRD pattern of trans-TTA-AzoDFP COF compared to the azobenzene free TTA-DFP COF could be explained by the distortion of the $\pi-\pi$ stacking between the layers in trans-TTA-AzoDFP. 
10. Azo-DFP monomer: ${ }^{1} \mathrm{H}$ NMR spectral analysis before and after UV-light irradiation in solution
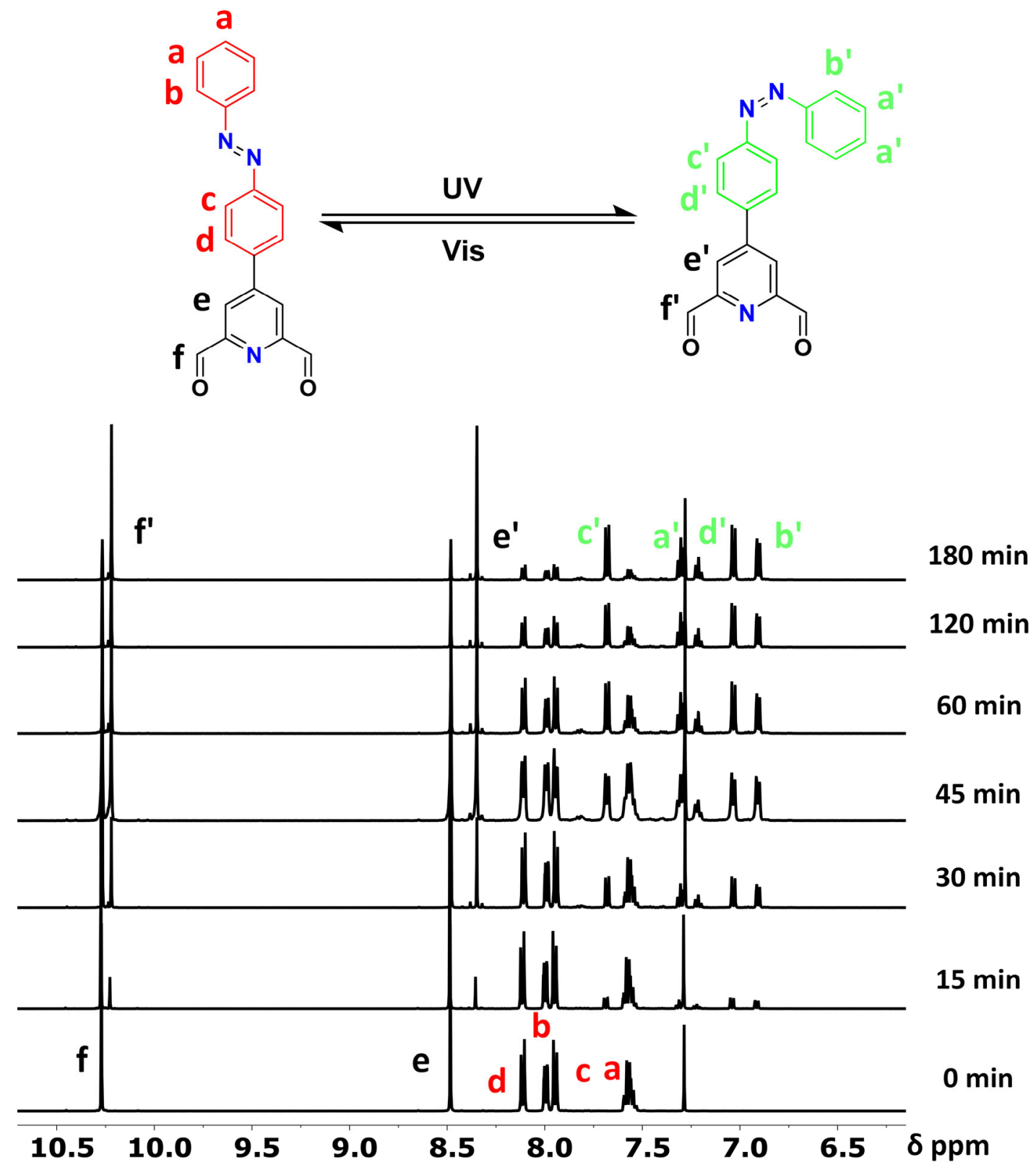

Figure S9: ${ }^{1} \mathrm{H}$ NMR (500 MHz) spectral changes of $(E)$-4-(4-(phenyldiazenyl)phenyl)pyridine2,6-dicarbaldehyde (Azo-DFP - $0.6 \mathrm{~mL}$ of $2.64 \mathrm{mM}$ ) upon irradiation with light (360 nm) for $15,30,45,60,120$ and 180 minutes in $\mathrm{CDCl}_{3}$ at $298 \mathrm{~K}$. After 180 minutes exposure to UV light, the trans form is converted into the cis form with a conversion ratio of 24:76 (trans:cis) and the percentage of conversion was calculated by integrating the areas of $\mathrm{H}_{\mathrm{f}}$ and $\mathrm{He}_{\mathrm{e}}$ of the cis and trans isomers proton signals using TMS as an internal standard. 


\section{Time dependent UV-Vis study of TTA-AzoDFP}

a)

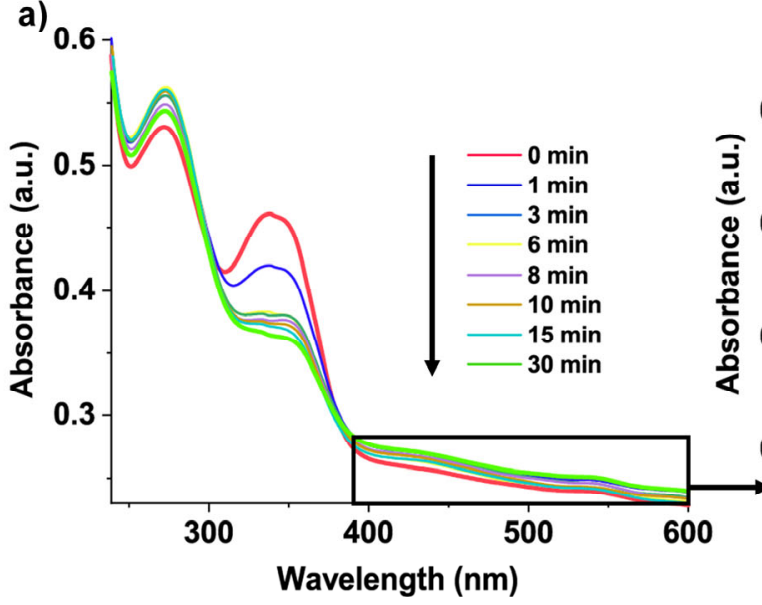

b)

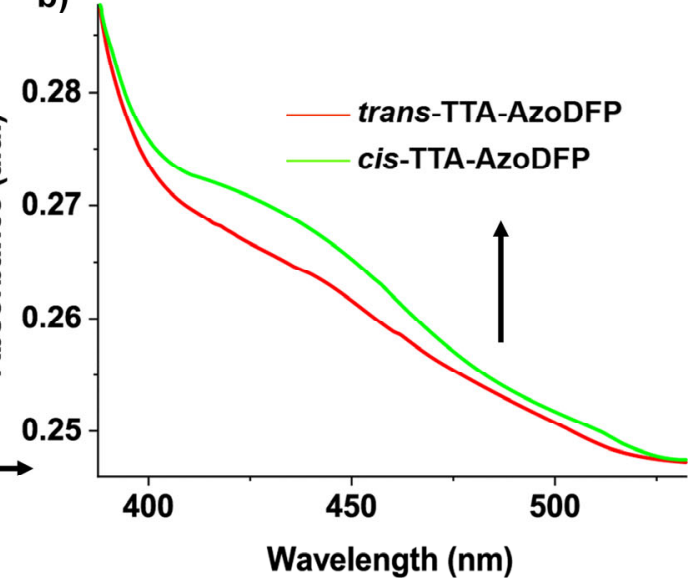

Figure S10: a) UV-Vis spectral changes of trans-TTA-AzoDFP dispersed in ethanol under UVlight $(365 \mathrm{~nm})$ irradiation at different time intervals; b) Zoomed UV-Vis spectra of TTAAzoDFP clearly indicates that a new band appears at $431 \mathrm{~nm}$, which corresponds to $\mathrm{n}-\pi^{*}$ transition. Adsorption experiments were performed at room temperature in a quartz cuvette $(l=$ $1 \mathrm{~cm}$ ) directly in the cell holder of a UV-Vis spectrophotometer under ambient conditions.

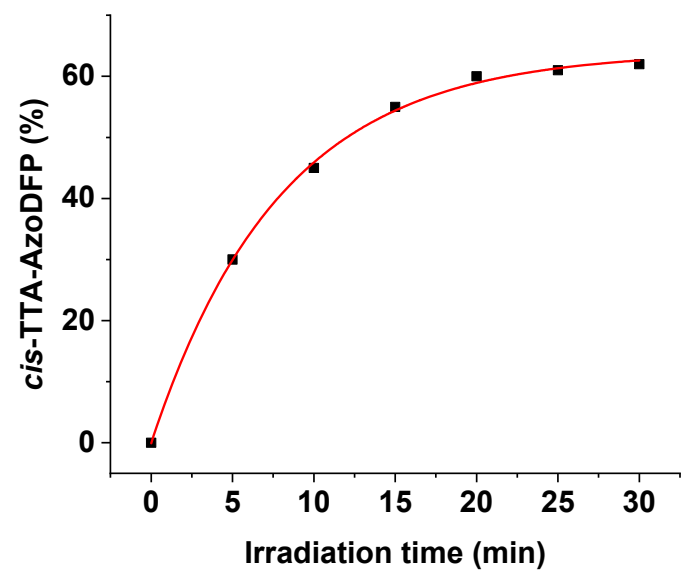

Figure S11: The non-linear fitting curves of trans-TTA-AzoDFP upon UV-light irradiation (365 nm).

The percentage of cis-TTA-AzoDFP isomer was calculated from the following equation:

$\%$ cis $=\frac{\text { Atrans }- \text { Acis }}{\text { Atrans }} * 100$

$\%$ cis $=$ Fraction of $c i s$-TTA-AzoDFP isomer upon UV-light $(365 \mathrm{~nm})$ irradiation on trans-TTA-AzoDFP dispersed in ethanol.

$\mathrm{A}_{\text {trans }}=$ Maximum absorbance of trans-TTA-AzoDFP.

$\mathrm{A}_{c i s}=$ Maximum absorbance of $c i s$-TTA-AzoDFP. 


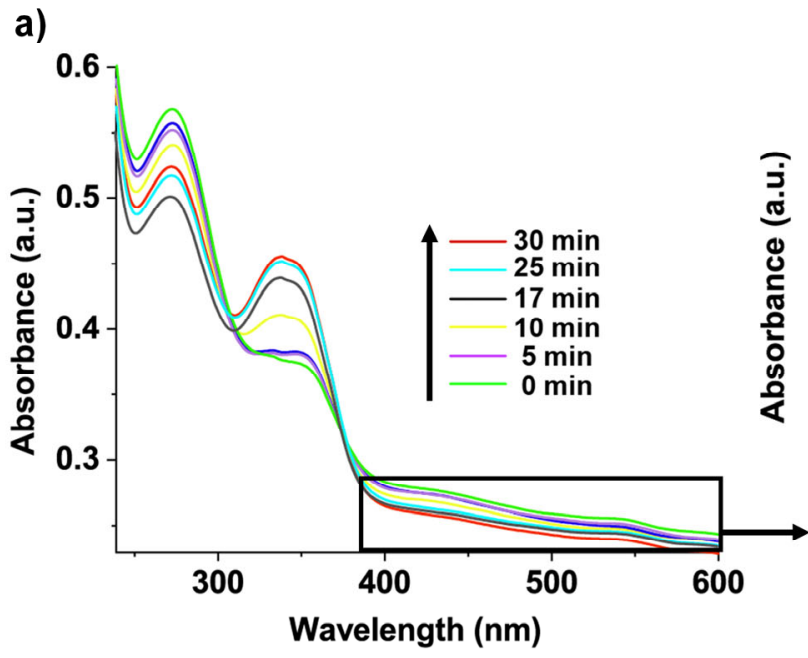

b)

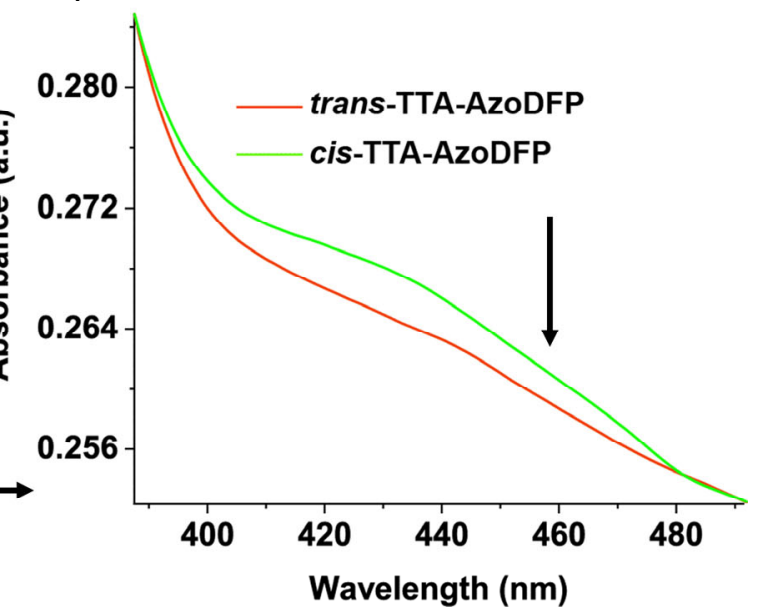

Figure S12: a) UV-Vis spectral changes of cis-TTA-AzoDFP dispersed in ethanol and irradiated with a visible-light (450 nm) as a function of time; b) Zoomed UV-Vis spectra of TTA-AzoDFP highlighting the decrease of the band corresponding to $n-\pi^{*}$ transition. Adsorption experiments were performed at room temperature in a quartz cuvette $(l=1 \mathrm{~cm})$ directly in the cell holder of a UV-Vis spectrophotometer under ambient conditions. 


\section{Photostability of TTA-AzoDFP}

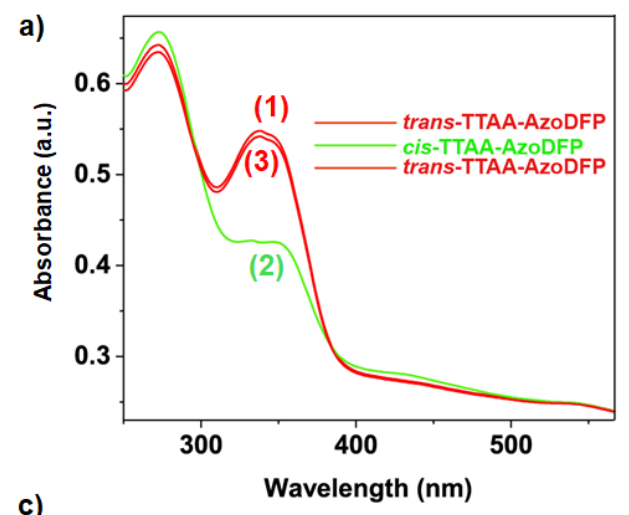

c)

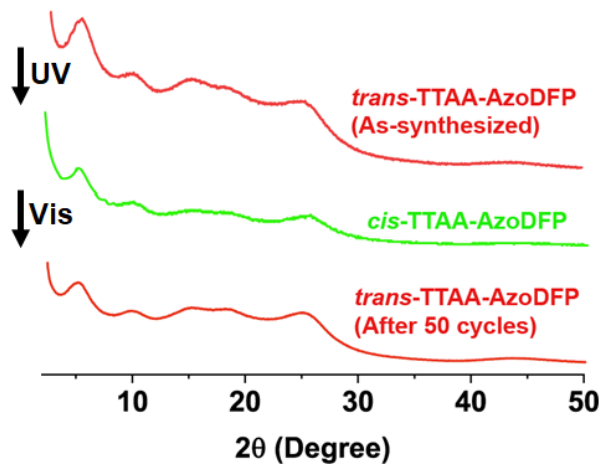

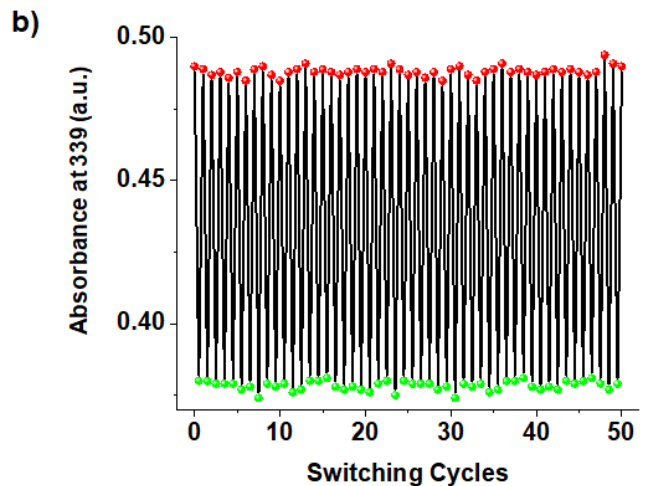

d)

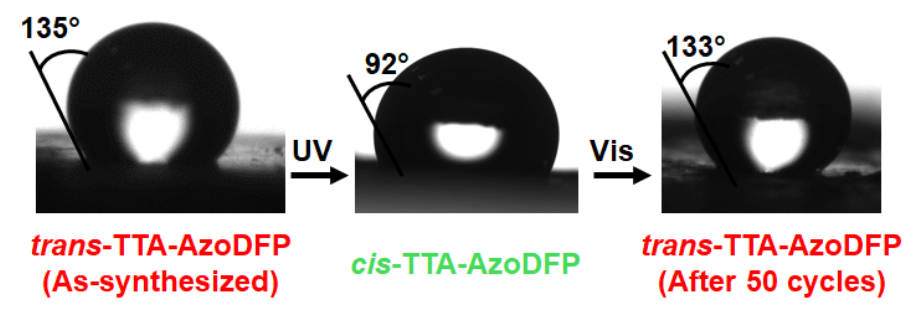

Figure S13: a) UV-Vis spectral changes of the isomerization of the as-synthesized trans-TTAAzoDFP (1) to cis- TTA-AzoDFP (2) dispersed in ethanol upon irradiation at $365 \mathrm{~nm}$ and the recovered UV-Vis spectrum of trans-TTA-AzoDFP (3, after 50 cycles) upon irradiation at 450 $\mathrm{nm}$; b) 50 photo-switching cycles of TTA-AzoDFP dispersed in ethanol at room temperature upon alternating the irradiation wavelength between 365 and $450 \mathrm{~nm}$ light; c) PXRD patterns of the as-synthesized trans-TTA-AzoDFP (red line) and cis-TTA-AzoDFP (green line) upon irradiation with a $365 \mathrm{~nm}$ light and the recovered PXRD pattern of trans-TTA-AzoDFP (red line, after 50 cycles) upon irradiation with a $450 \mathrm{~nm}$ light; d) Contact angle (CA) measurements of the as-synthesized trans-TTA-AzoDFP $\left(\mathrm{CA}=135^{\circ}\right)$, the cis- TTA-AzoDFP $\left(\mathrm{CA}=92^{\circ}\right)$ upon irradiation with the $365 \mathrm{~nm}$ light for 15 minutes and the recovered contact angle of trans-TTAAzoDFP $\left(\mathrm{CA}=133^{\circ}\right.$, after 50 cycles $)$ upon irradiation with the $450 \mathrm{~nm}$ light for 15 minutes. 
trans-TTA-AzoDFP

(As-synthesized)
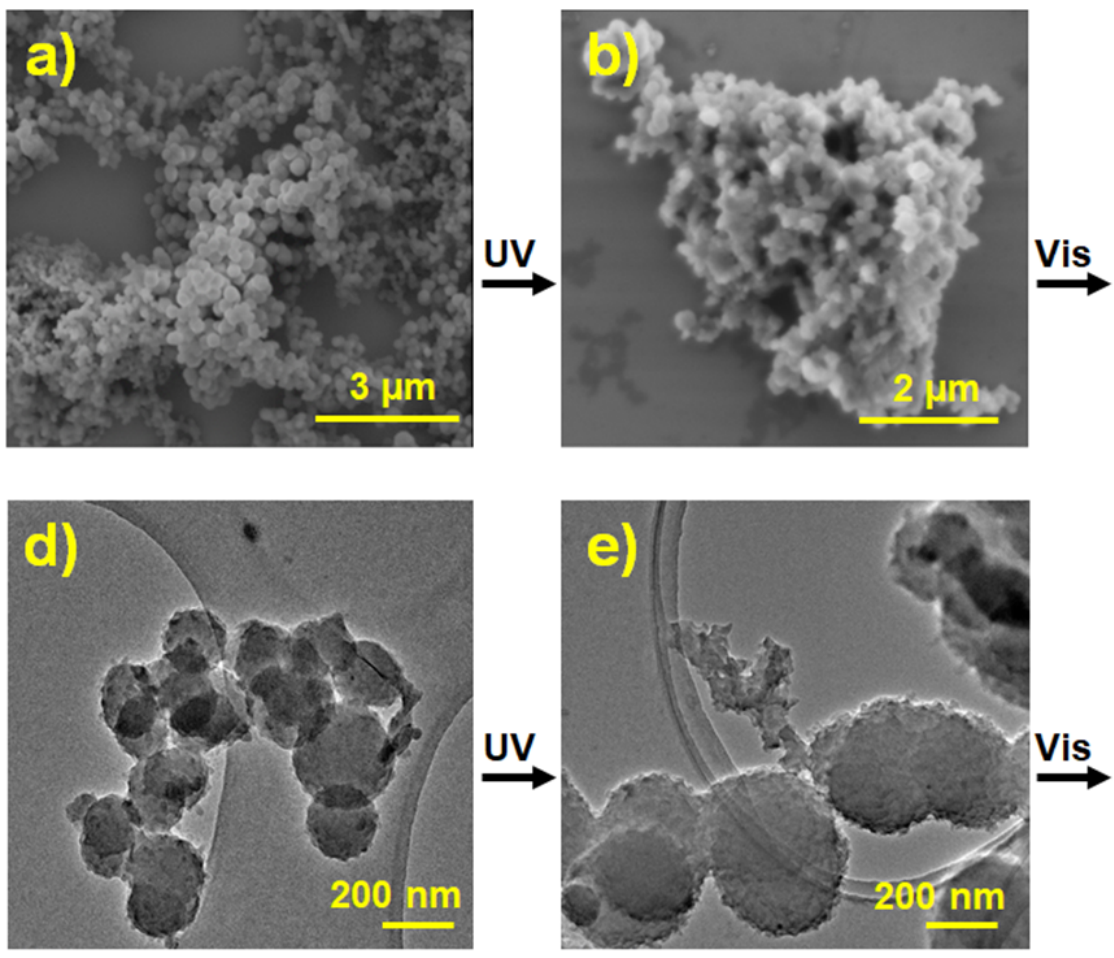

trans-TTA-AzoDFP

(After 50 cycles)
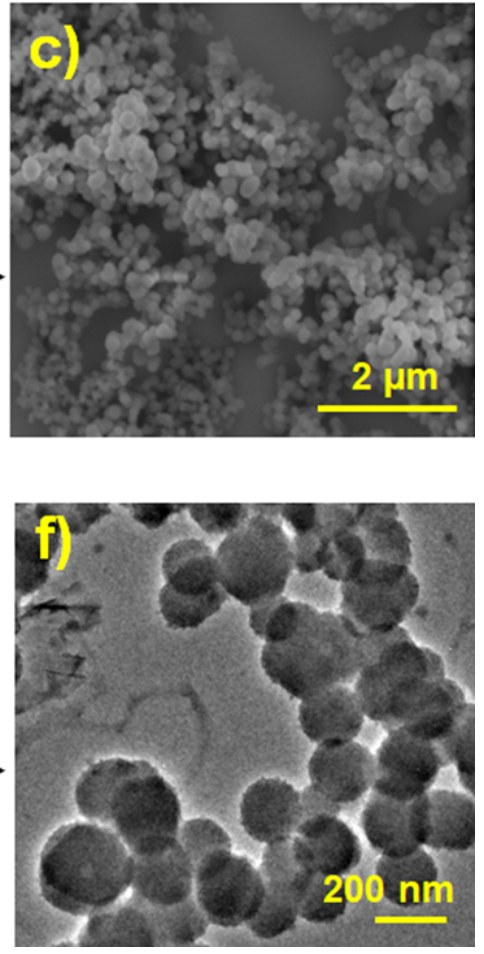

Figure S14: Microscopic characterization. SEM (a-c) and HRTEM (d-f) images showing the morphological changes of the as-synthesized trans-TTA-AzoDFP (a, d) upon irradiation with a $365 \mathrm{~nm}$ UV-light to cis- TTA-AzoDFP (b, e). The recovered SEM and HRTEM images (c, f) of TTA-AzoDFP after alternating the irradiation wavelength between $365 \mathrm{~nm}$ and $450 \mathrm{~nm}$ lights for 50 cycles. The maintained morphology after fifty cycles confirms the photostability of TTAAzoDFP. 
13. Luminescence study of Azo-DFP

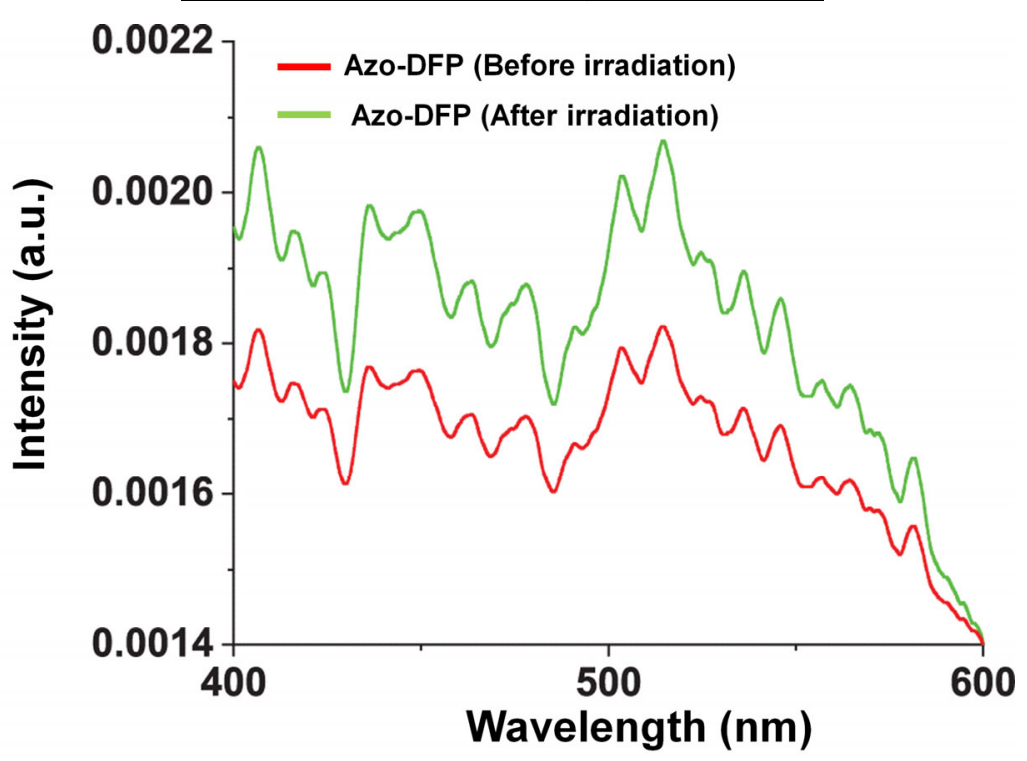

Figure S15: Luminescent spectra of the precursor Azo-DFP in ethanol solution $\left(10^{-5} \mathrm{M}\right)$ before and after irradiation with UV-light ( $365 \mathrm{~nm}, 30 \mathrm{~min}$ ) using an excitation wavelength of $339 \mathrm{~nm}$, under ambient conditions. The low quality of the spectra is the result of the very weak emission of Azo-DFP.

\section{BET analysis of TTA-AzoDFP COF}

a)

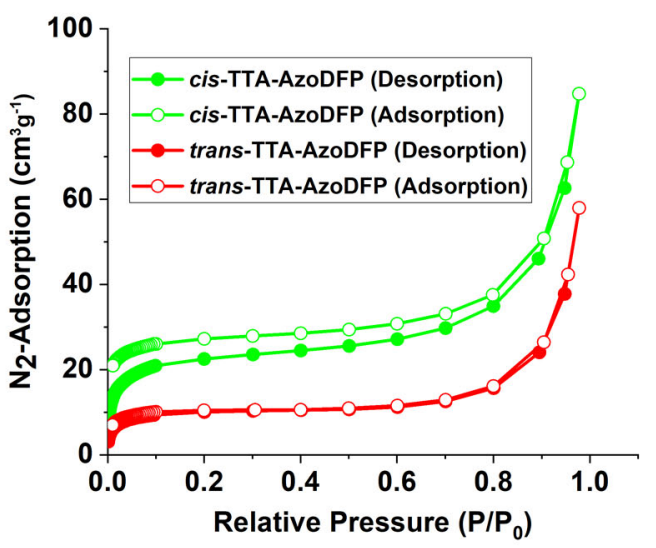

b)

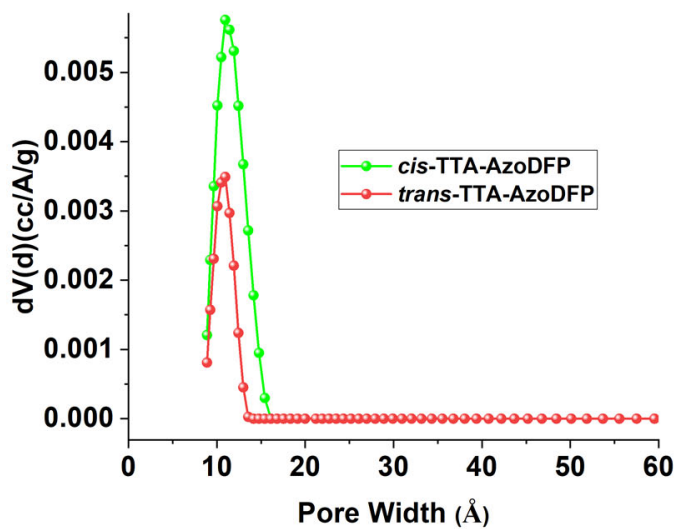

Figure S16: a) $\mathrm{N}_{2}$-Adsorption isotherms and b) pore size distributions of trans-TTA-AzoDFP (red) and cis-TTA-AzoDFP (green). BET analysis of cis-TTA-AzoDFP was carried out by exposing the solid sample (trans-TTA-AzoDFP) to a UV-light ( $365 \mathrm{~nm}$ ) for 5 hours and analysis was performed immediately. BET analysis was performed by activating the sample at $80{ }^{\circ} \mathrm{C}$ for 12 hours. 


\section{BET analysis of TTA-DFP COF}
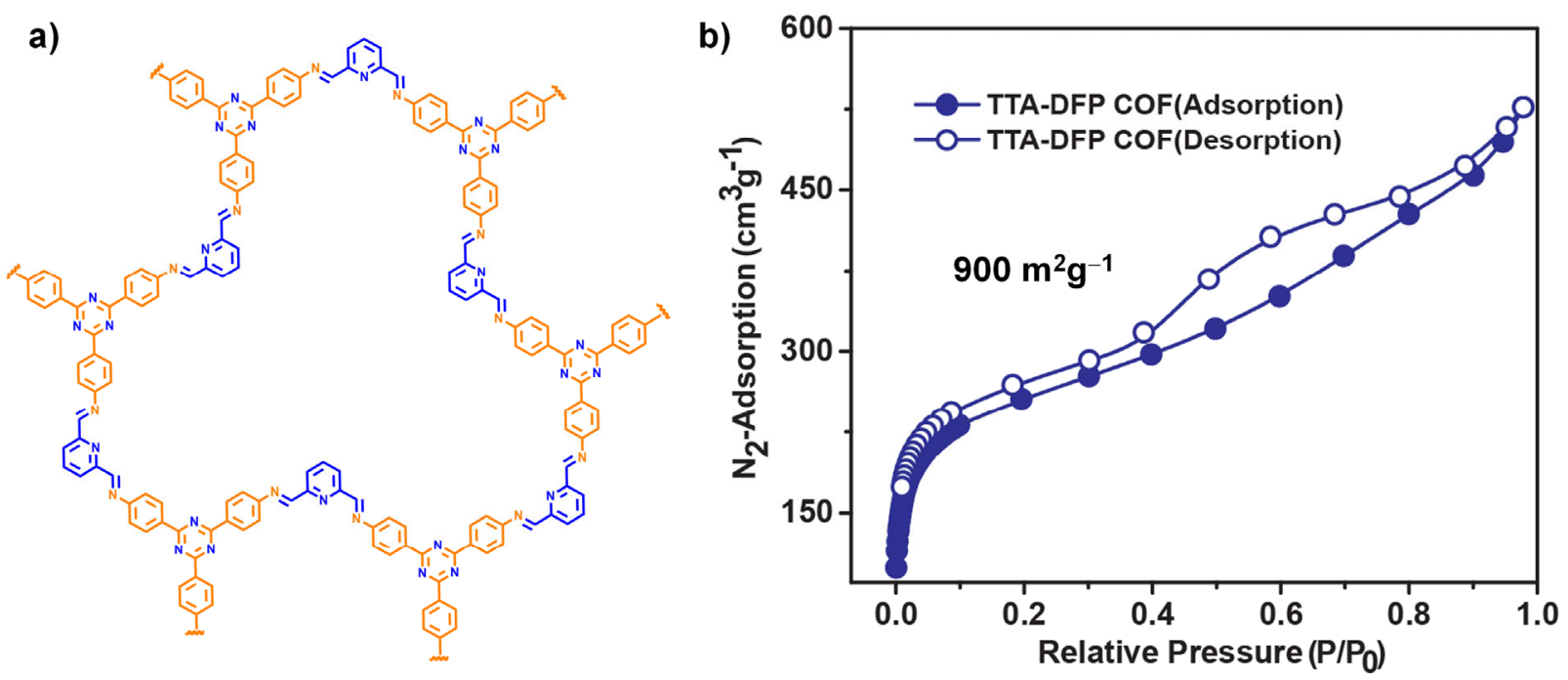

Figure S17: a) Chemical structure of the azobenzene-free TTA-DFP COF and b) $\mathrm{N}_{2}$-Adsorption isotherms of TTA-DFP COF, synthesized under solvothermal conditions.

16. Solid state ${ }^{13} \mathrm{C}$ cross-polarization magic-angle spinning NMR spectral analysis before and after UV-light irradiation

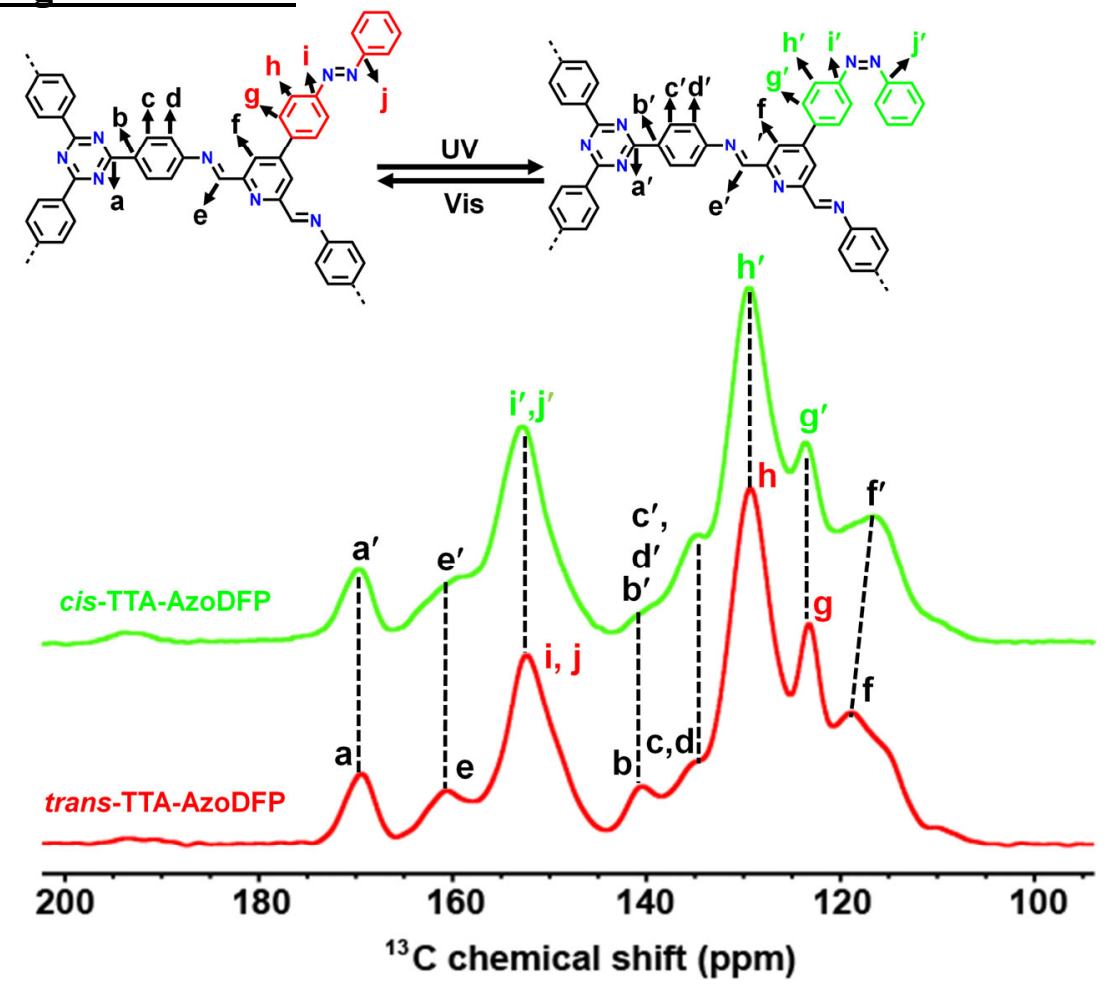

Figure S18: Solid-state ${ }^{13} \mathrm{C}$ CP-MAS NMR analysis of trans-TTA-AzoDFP (red) and cis-TTA-AzoDFP (green). The NMR spectrum of cis-TTA-AzoDFP was recorded upon irradiation of $\sim 50 \mathrm{mg}$ solid transTTA-AzoDFP with UV-light (365 nm) under ambient conditions for 1 hour. 


\section{DLS experiment of the photo-isomerization of trans-TTA-AzoDFP}

a)

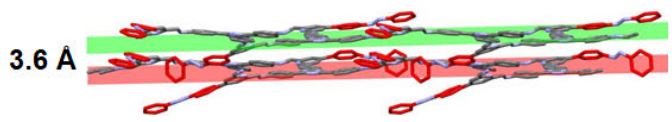

b)

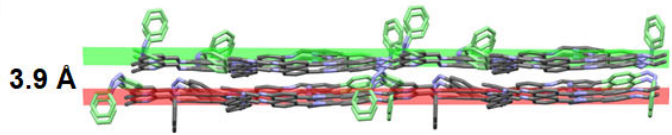

c)

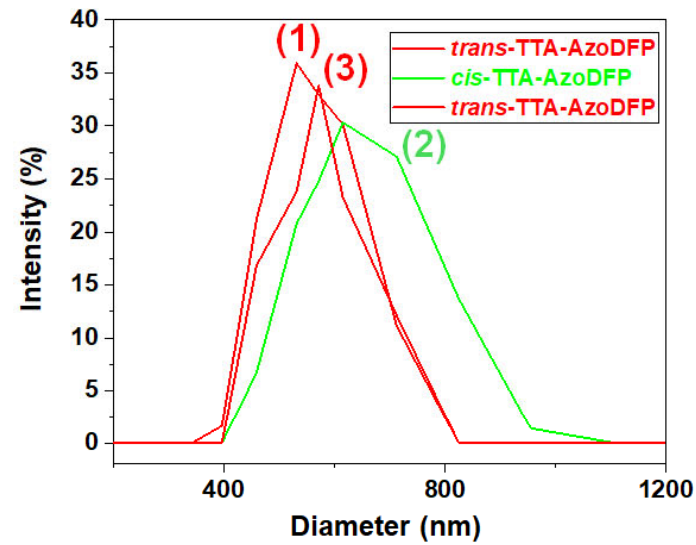

d)

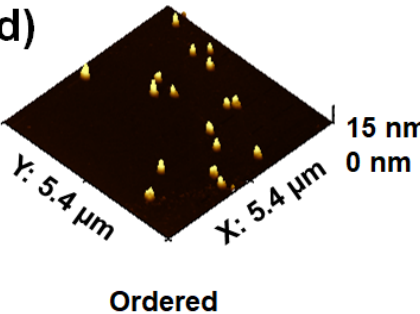

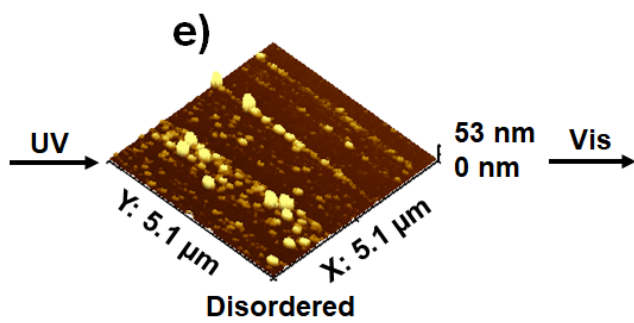

Disordered

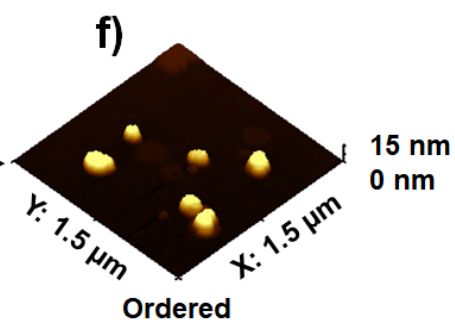

Figure S19: Side view of ball and stick representation of four stacked layers of trans-TTA-AzoDFP (a) and cis-TTA-AzoDFP (b), the inter layer spacing distances were calculated by considering the distance between the two layers; c) DLS experiment showing the change in the average size of trans-TTAAzoDFP (1) dispersed in ethanol at room temperature upon isomerization to cis-TTA-AzoDFP dispersed in ethanol at room temperature upon irradiation at $365 \mathrm{~nm}$, and the recovered DLS spectra of trans-TTA-AzoDFP (3) upon irradiation at $450 \mathrm{~nm}$.; The changes of AFM surface topology upon UV irradiation at room temperature of trans-TTA-AzoDFP before illumination (d), upon irradiation with 365 $\mathrm{nm}$ UV light (e) and re-irradiation with $450 \mathrm{~nm}$ light (f). 
18. Light-triggered capture and release of Rhodamine B and theortical calculations of RhB within the structure.

a)

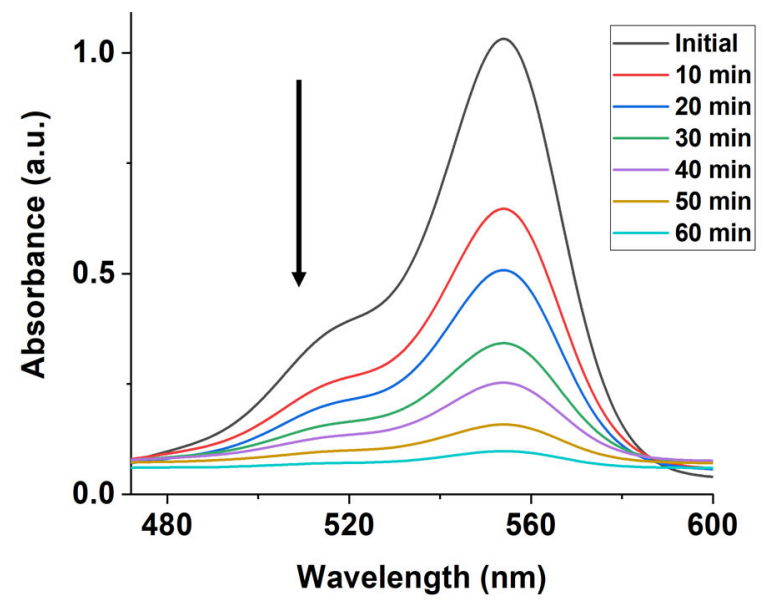

b)

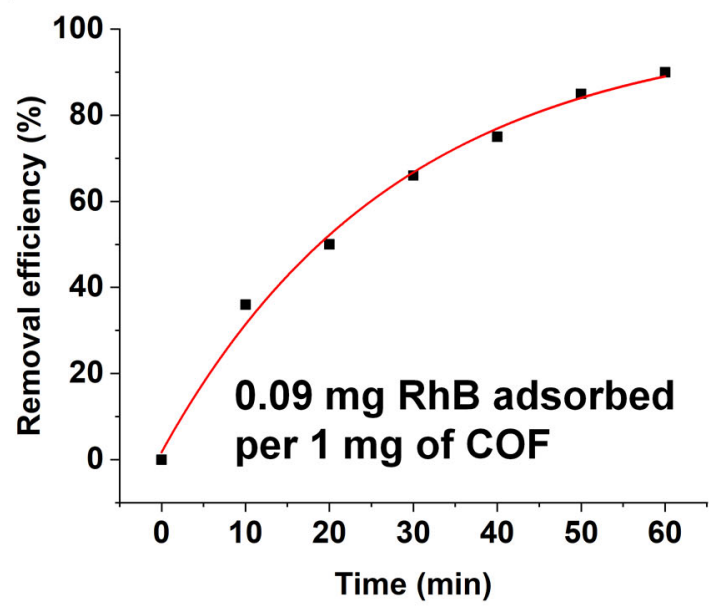

Figure S20: a) UV-Vis absorbance spectra of an aqueous solution of RhB in the presence of 1 mg of trans-TTA-AzoDFP at different time intervals; b) Removal efficiency of RhB by transTTA-AzoDFP over 60 minutes. Condition: $\sim 1 \mathrm{mg}$ of trans-TTA-AzoDFP was immersed in 2 $\mathrm{mL}$ deionized water containing $2 \times 10^{-5} \mathrm{M} \mathrm{RhB}$ and the spectra were recorded at room temperature in a $1 \mathrm{~cm}$ Quartz cuvette.

\section{Amount of RhB can be calculated from following equation}

$\mathrm{Q}_{\mathrm{t}}=\left(\mathrm{C}_{0}-\mathrm{C}_{\mathrm{t}}\right) \mathrm{V} / \mathrm{m}$

$\mathrm{Q}_{\mathrm{t}}, \mathrm{C}_{0}, \mathrm{C}_{\mathrm{t}}, \mathrm{V}$ and $\mathrm{m}$ are the amount of $\mathrm{RhB}$ adsorbed, the initial concentration of the $\mathrm{RhB}$ solution, the concentration of the RhB solution at specific times, the volume of the solution and the mass used for the $\mathrm{RhB}$ respectively. 


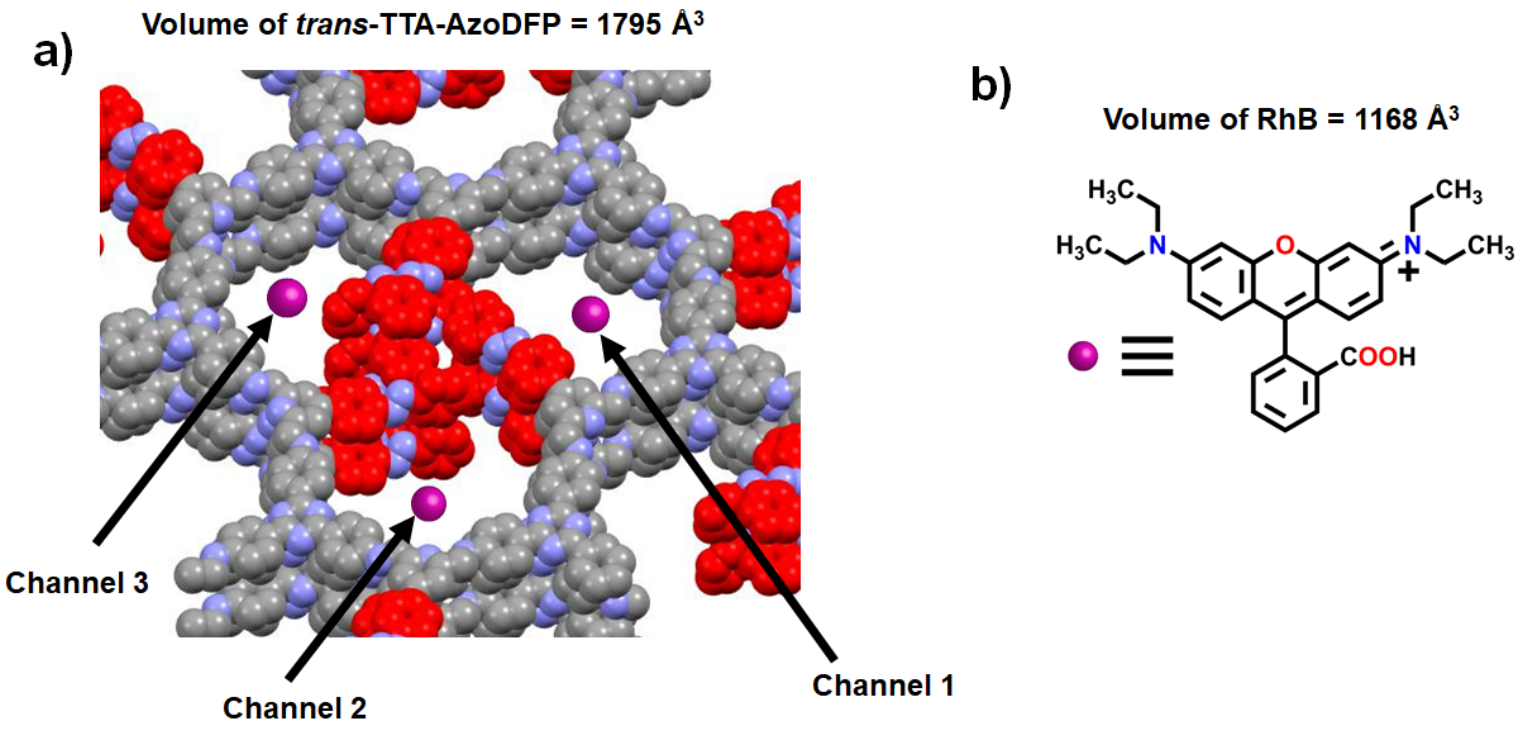

Considering $2 \times 2 \times 2$ unit cell structure, containing 4 layers.

If 2 unit cell structure take 3 molecules of $R h B$, then one unit cell will take ( $3 / 2=1.5$ molecules of $R h B)$.

Figure S21: a) Two-unit cell packing diagram of trans-TTA-AzoDFP showing that three molecules of RhB can fit into each channel as shown in the figure; b) Chemical structure of $\mathrm{RhB}$ with actual occupying volume.

\section{BET analysis after loading of $\mathrm{RhB}$}

a)

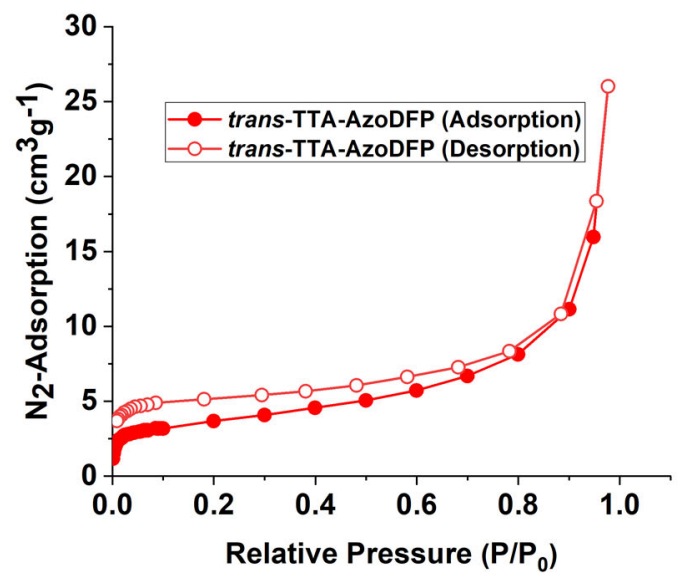

b)

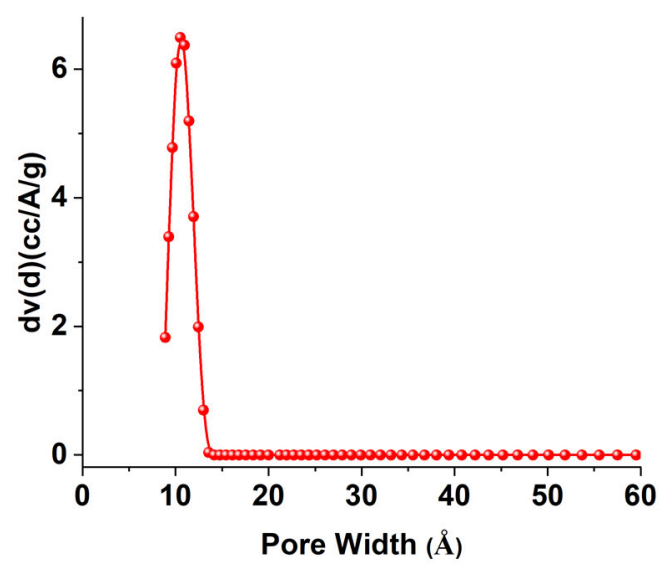

Figure S22: $\mathrm{N}_{2}$-Adsorption isotherm of RhB-loaded trans-TTA-AzoDFP COF. 

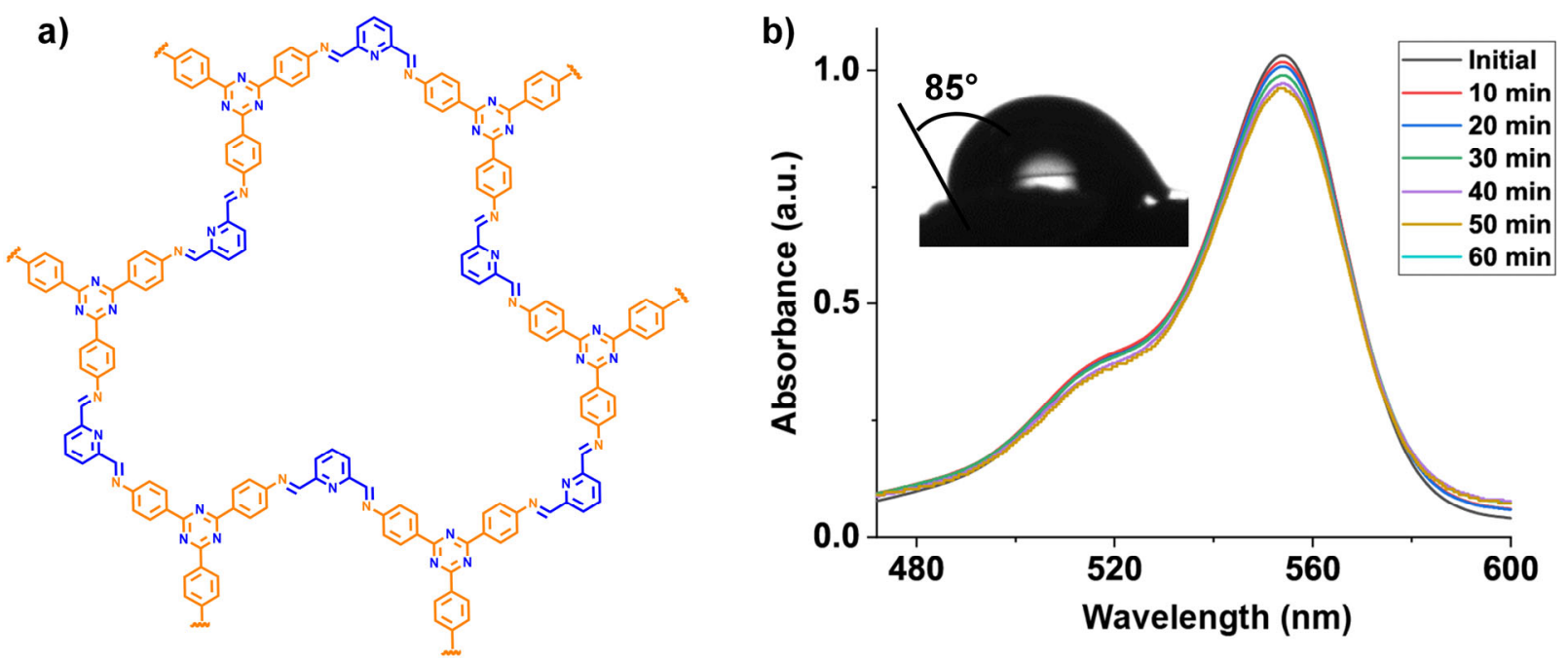

Figure S22: a) chemical structure of the azobenzene-free TTA-DFP COF; (b) UV-Vis spectra of $\mathrm{RhB}$ in presence of TTA-DFP showing low affinity for RhB. In UV-Vis experiment, $2 \times 10^{-}$

${ }^{5} \mathrm{M} \mathrm{RhB}(2 \mathrm{~mL})$ aqueous solution of $\mathrm{RhB}$ was stirred with $1 \mathrm{mg}$ of azo-benzene free COF for 60 min. TTA-DFP was removed by centrifugation. Conditions: $\sim 1 \mathrm{mg}$ TTA-AzoDFP was, immersed in $2 \mathrm{~mL}$ deionized water containing $2 \times 10^{-5} \mathrm{M} \mathrm{RhB}$ and the spectra were recorded at ambient temperature. Inset: Water contact angle of azobenzene-free TTA-DFP COF. The contact angle measurement for TTA-DFP COF was carried out by placing a water droplet on the TTADFP COF coated on a glass surface. 


\section{UV-Vis experiment of cargo release in response to UV-light}

$1 \mathrm{mg}$ of the RhB-loaded trans-TTA-AzoDFP COF was dispersed in $2 \mathrm{~mL}$ of deionized water at room temperature. The solution was transferred into a quartz cuvette $(l=1 \mathrm{~cm})$ and the UV spectrum was recorded at $20^{\circ} \mathrm{C}$. The solution was then irradiated with a $365 \mathrm{~nm}$ light for various interval time (as shown in Figure S23) with constant stirring. The corresponding UV spectra were recorded at $20^{\circ} \mathrm{C}$.



Figure S23: UV-visible spectra of the RhB solution after UV irradiation (365 nm) for different time intervals.

\section{Control UV-Vis experiment of cargo release in response to heat}

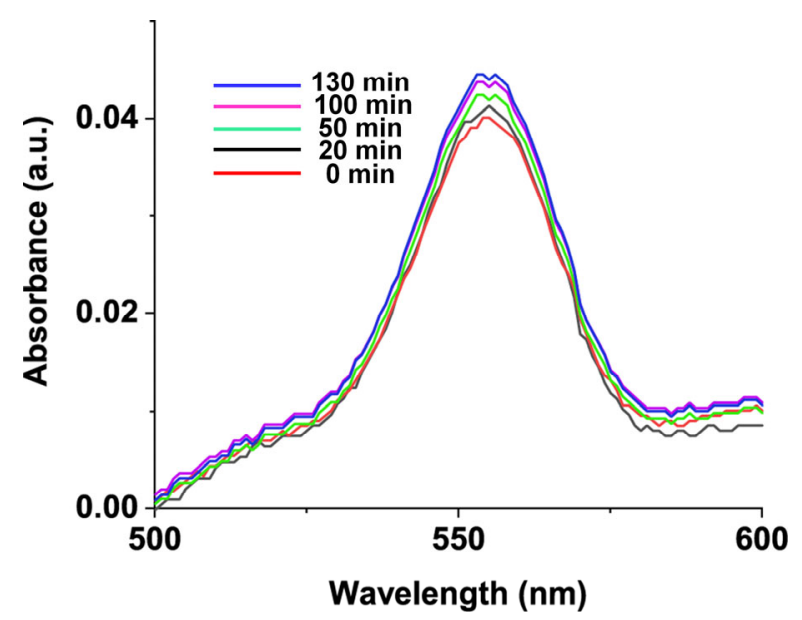

Figure S24: UV-visible spectra of the aqueous solution containing trans-TTA-AzoDFP@RhB after heating for different time. Conditions: $\sim 1 \mathrm{mg}$ of the RhB-loaded trans-TTA-AzoDFP was immersed in $2 \mathrm{~mL}$ deionized water and heated at $80{ }^{\circ} \mathrm{C}$ and at $0,20,50,100$ and 130 minute time interval the UV-Vis spectra were recorded. 


\section{BET analysis after regeneration}

a)

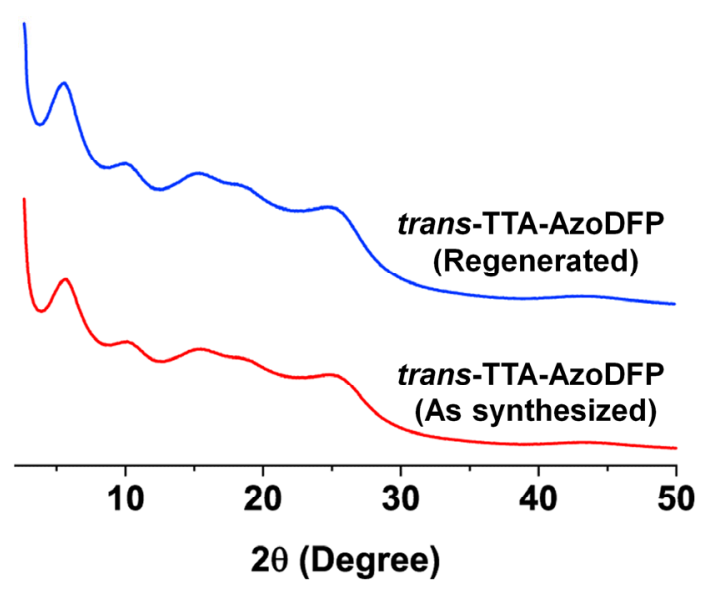

b)

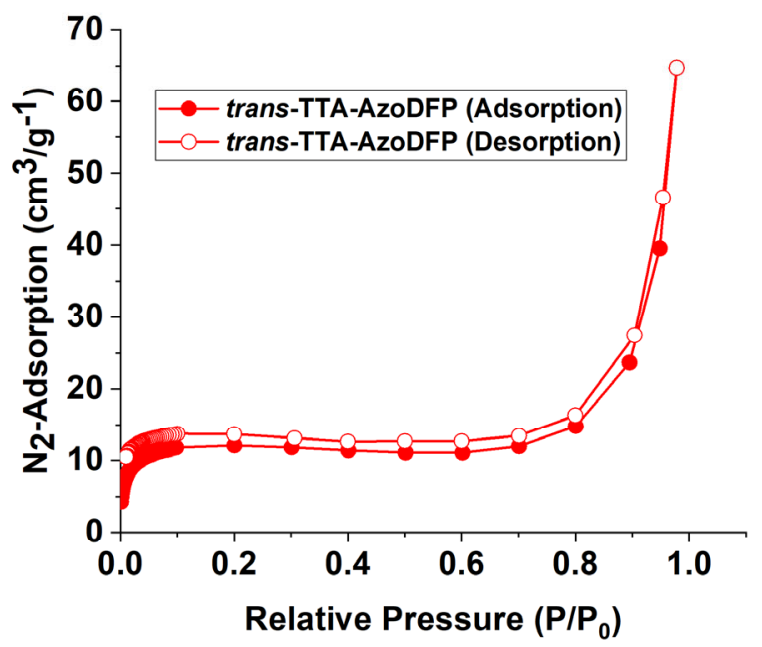

Figure S25: a) PXRD-pattern and $\mathrm{N}_{2}$-Adsorption isotherm of trans-TTA-AzoDFP COF after regeneration. After the release experiments was completed, the solid COF was collected from the solution by ultracentrifugation, dried for $12 \mathrm{~h}$ in oven at $120^{\circ} \mathrm{C}$. The isolated solid powder was exposed to visible light for five hours before recording the BET. 


\section{References}

1. Aricó, F.; Chang, T.; Cantrill, S. J.; Khan, S. I.; Stoddart, J. F., Template-Directed Synthesis of Multiply Mechanically Interlocked Molecules Under Thermodynamic Control. Chem.-Eur. J. 2005, 11 (16), 4655-4666.

2. Chichak, K. S.; Peters, A. J.; Cantrill, S. J.; Stoddart, J. F., Nanoscale borromeates. J. org. chem. 2005, 70 (20), 7956-7962.

3. Kallweit, C.; Haberhauer, G.; Woitschetzki, S., 4, 4'-Bipyridine as a Unidirectional Switching Unit for a Molecular Pushing Motor. Chem.- Eur. J. 2014, 20 (21), 63586365.

4. Gottlieb, H. E.; Kotlyar, V.; Nudelman, A., NMR chemical shifts of common laboratory solvents as trace impurities. J. org. chem. 1997, 62 (21), 7512-7515.

5. Fulmer, G. R.; Miller, A. J.; Sherden, N. H.; Gottlieb, H. E.; Nudelman, A.; Stoltz, B. M.; Bercaw, J. E.; Goldberg, K. I., NMR chemical shifts of trace impurities: common laboratory solvents, organics, and gases in deuterated solvents relevant to the organometallic chemist. Organometallics 2010, 29 (9), 2176-2179.

6. Halder, A.; Kandambeth, S.; Biswal, B. P.; Kaur, G.; Roy, N. C.; Addicoat, M.; Salunke, J. K.; Banerjee, S.; Vanka, K.; Heine, T., Decoding the morphological diversity in two dimensional crystalline porous polymers by core planarity modulation. Angew. Chem. Int. Ed. 2016, 55 (27), 7806-7810.

7. Lu, X.; Gaus, M.; Elstner, M.; Cui, Q., Parametrization of DFTB3/3OB for Magnesium and Zinc for Chemical and Biological Applications. J. Phy. Chem. B 2015, 119 (3), 10621082.

8. Kubillus, M.; Kubař, T.; Gaus, M.; Řezáč, J.; Elstner, M., Parameterization of the DFTB3 Method for $\mathrm{Br}, \mathrm{Ca}, \mathrm{Cl}, \mathrm{F}, \mathrm{I}, \mathrm{K}$, and $\mathrm{Na}$ in Organic and Biological Systems. J. Chem. Theory Comput. 2015, 11 (1), 332-342.

9. Lukose, B.; Kuc, A.; Heine, T., The Structure of Layered Covalent-Organic Frameworks. Chem.-Eur. J. 2011, 17 (8), 2388-2392. 